This manuscript is a non-peer reviewed preprint that has been submitted for publication. Subsequent versions of this manuscript may have updated content. Feedback and comments are welcomed, feel free to contact the corresponding author:

Alexandre Wadoux

alexandre.wadoux@sydney.edu.au 


\title{
Machine learning for digital soil mapping: applications, challenges and suggested solutions
}

\author{
Alexandre M.J-C. Wadoux ${ }^{\mathrm{a}, *}$, Budiman Minasny ${ }^{\mathrm{a}}$, Alex B. McBratney ${ }^{\mathrm{a}}$ \\ ${ }^{a}$ Sydney Institute of Agriculture 83 School of Life and Environmental Sciences, The University of \\ Sydney, Australia
}

\begin{abstract}
The uptake of machine learning (ML) algorithms in digital soil mapping (DSM) is transforming the way soil scientists produce their maps. Machine learning is currently applied to mapping soil properties or classes much in the same way as other unrelated fields of science. Mapping of soil, however, has unique aspects which require adaptations of the ML algorithms. These features are for example, but not limited to, the inclusion of pedological knowledge into the ML algorithm, the accounting of spatial structure present in the soil data, or the desire to increase our scientific understanding of the distribution and genesis of soil from a calibrated ML model. Tackling these challenges is critical for machine learning to gain credibility and scientific consistency in soil science. In this article, we review the current applications of machine learning in digital soil mapping and suggest improvements. We found a growing interest of the use of ML in DSM. Most studies focus on obtaining accurate maps and disregard the characteristics of soil data, such as spatial autocorrelation. Only a few studies account for existing soil knowledge or quantify the uncertainty of the predicted maps. We then discuss the challenges related to the application of ML for soil mapping and offer solutions from existing studies in the natural sciences. The challenges are organized as follows: sampling, resampling, accounting for the spatial information, multivariate mapping, uncertainty analysis, validation, integration of pedological knowledge and, interpretation of the models. We conclude that for future developments, machine learning should incorporate three core elements: plausibility, interpretability, and explainability, which will trigger soil scientists to move beyond model prediction and towards explanation of soil processes.
\end{abstract}

${ }^{*}$ Corresponding author: Sydney Institute of Agriculture \& School of Life and Environmental Sciences, The University of Sydney, New South Wales, Australia

Email address: alexandre.wadoux@sydney.edu.au (Alexandre M.J-C. Wadoux)

Preprint (Review article)

February 6, 2020 
Keywords: Soil science, Pedometrics, Data mining, Spatial data, Geostatistics, Random forest

\section{Introduction}

In recent years, soil science has witnessed a considerable increase in digital soil mapping activities. This is caused by the convergence of several timely factors which are, among others, a huge demand for quantitative and spatial soil information, the accumulation of databases of measured or inferred soil properties coupled with exhaustively known environmental variables and the development of numerical models combined with computer resources to mine these stores of soil data. The digital soil mapping (DSM) framework was formalized by the publication of McBratney et al. (2003) which builds on Jenny's $S=$ clorpt model (Jenny, 1941) of soil formation, where $S$ is the soil and the acronym clorpt stands for climate, organisms, relief, parent material and time, respectively. In short, clorpt is a list of variables which, if they are known without error, are likely to explain the soil variation over a region. McBratney et al. (2003) supplemented Jenny's formulation with $n$, which stands for spatial position, and advocated the scorpan model for soil spatial variation. This updated equation provides a spatial model to express quantitatively the relationship between a soil property or class and environmental variables, for a given spatial location.

Conventionally, spatial prediction of soil has been embedded in the geostatistical framework (Heuvelink \& Webster, 2001) in which a sample of a soil property is modelled as a sum of a linear combination of environmental covariates and a spatially autocorrelated (stochastic) residual, and prediction at unobserved locations is made by kriging. Geostatistical models are often used in soil mapping because they have several advantages (Oliver, 1987). First, a statistically sound model is assumed for spatial variation. This enables interpretation of the underlying physical processes conveyed (inferred) by the model. Secondly, spatial autocorrelation is explicitly modelled. This is relevant for environmental variables such as soil which vary from place to place, but exhibit correlation between places. Thirdly, an explicit measure of the uncertainty is associated with the prediction. In many circumstances such as in a decision making process, the prediction is not the only interest and uncertainty maps are required for the evaluation of the map quality or modelling risk.

Geostatistical mapping of soil has, conversely, several limitations which have only partially been resolved in the current literature. To begin, the residuals are as- 
sumed normally distributed, stationary (with constant mean and unit variance) and isotropic. Next, modelling the non-linear relation between a soil property or class and numerous cross-correlated covariates is not straightforward and introduces additional challenges (e.g. many parameters have to be estimated). Finally, geostatistical models are computationally demanding if the sample size and/or the number of prediction locations are large (Cressie \& Johannesson, 2008).

As an alternative, machine learning (ML) emerged in the 1990s as a tool for spatial prediction and digital soil mapping (Lagacherie, 2008). Machine learning techniques refer to a large class of non-linear data-driven algorithms employed primarily for data mining and pattern recognition purposes, and now frequently used for regression and classification tasks in all fields of science. ML algorithms do not make an assumption of the observations' distribution, unlike geostatistical methods where transformation of the original observations is often required to satisfy the assumptions. ML algorithms can also handle a large number of cross-correlated covariates as predictor.

In parallel, there has been a tremendous increase in the production and availability of regional and global soil databases. For example, the Soil and Terrain Digital Database (SOTER, Oldeman \& Van Engelen (1993)) made by FAO-UNESCO compiled quantitative information on soil and terrain for different parts of the world while WoSIS is a harmonised database of more than 6 million geo-referenced soil records (Batjes et al., 2017). Additionally, numerous spatially exhaustive scorpan covariates are available at global scale for climate (Fick \& Hijmans, 2017), elevation (Yamazaki et al., 2017), and parent material (Hartmann \& Moosdorf, 2012). Further potential covariates are provided by remote sensing such as by the MODIS (Mira et al., 2015) satellite or Sentinel-2A hyperspectral sensor (Gascon et al., 2017). Soil mappers are now confronted with an increasing complexity in both soil data and covariates. Conventional regression techniques seem, to some extent, outdated to accommodate the increased complexity of soil datasets. This justifies the increasing use of machine learning algorithms for digital soil mapping.

An essential distinction between conventional (statistical and geostatistical) models and ML algorithms applied in DSM is their purpose. Machine learning algorithms mostly emphasize prediction accuracy whereas statistical models infer the process which generated the data through a pre-defined model of spatial variation. In the latter case, any interpretation is made in light of the model functions and the value of the covariates or input data. In machine learning, a predictive model is constructed 
to predict a set of input values to output values using an error-minimization procedure. Since ML algorithms are not conditioned to follow any statistical assumptions, they often appear more accurate than conventional models. The exact path between input and output is ignored, and may not resemble an actual process described by the existing knowledge. In soil science, the explosion of articles using ML algorithms have made difficult to see the difference between model fitting and inference, and, as a result between data science and soil science. Research seems to be driven by the technique rather than by the hypothesis to be tested. This seems a poor bet for the advancement of knowledge since "almost invariably the technician's skill is a solution looking for a problem" (Braben, 1985).

In DSM, the use of ML algorithms has led to an increasing number of publications where prediction (viz. mapping) of a soil property or class is the main interest. Many "easy-to-follow" software implementations have supported this increase. Digital soil mapping, however, has unique characteristics which require adaptation of the ML algorithms. These features are for example, but not limited to, the inclusion of pedological knowledge in the ML algorithm, the accounting of spatial structure present in the raw soil data, or the need to increase our scientific understanding of the soil from a calibrated ML model.

This article aims to review the development of ML applied to digital soil mapping by identifying key challenges and opportunities to solve them from the literature. In this review, we define ML as the computer assisted practice of using data-driven (and mostly non-linear) algorithms which resort to a large amount of calibration data to learn a pattern and make a prediction. We start by reviewing and summarizing the current use of machine learning in DSM. Based on this summary, we identify gaps in the knowledge and define areas in which adapting ML algorithms would be beneficial for their use in DSM. We propose solutions and a framework based on the literature from different fields of natural science. Finally, we define three core elements that should trigger soil scientists to move from model prediction to explanation of soil processes.

\section{A summary of applications}

\subsection{Extent, resolution, depths}

Table 1 summarizes some recent case studies of digital soil maps that have been produced using a ML algorithm. There is a large range of case studies, mapping soil properties or classes from the plot $\left(<1 \mathrm{~km}^{2}\right)$ to the global $\left(>10^{7} \mathrm{~km}^{2}\right)$ scale. Most 
studies in our literature review predict at a local to regional scale. The mean extent of the study area is $3,900 \mathrm{~km}^{2}$, but most (90\%) studies consider a study area smaller than $650,000 \mathrm{~km}^{2}$ (equivalent to the size of metropolitan France). Few studies map at plot or global scales. For example, Pouladi et al. (2019) make a quantitative map over a 10 ha $\left(0.1 \mathrm{~km}^{2}\right)$ field in Denmark while Hengl et al. (2017a) produce quantitative and categorical maps for the whole world.

We found a clear correlation between the spatial extent of the study area and the grid spacing (i.e. the spacing between point predictions) at which the soil property or class is mapped: the larger the study area, the coarser the resolution. The resolution spans between $2 \mathrm{~m} \times 2 \mathrm{~m}$ (Lacoste et al., 2014) to $1 \mathrm{~km} \times 1 \mathrm{~km}$ for large, regional or continental study areas (e.g. Hengl et al., 2014). Most studies, however, map at a standard spatial resolution of 30,90 or $250 \mathrm{~m}$.

While most of the studies (70\%) predict a soil property or class for a single depth (topsoil), a number of studies accounts for the soil variation at multiple depths. Viscarra-Rossel et al. (2015) follow the GlobalSoilMap project specifications (Arrouays et al., 2014) to produce a quantitative three dimensional map of several soil properties for six depths intervals, namely $0-0.05 \mathrm{~m}, 0.05-0.15 \mathrm{~m}, 0.15-0.30 \mathrm{~m}, 0.30$ $0.60 \mathrm{~m}, 0.60-1.00 \mathrm{~m}$ and 1.00-2.00 m. Similar depth intervals are used in Mulder et al. (2016) and Adhikari et al. (2014) for soil organic prediction in France or Denmark, respectively. Several other studies (e.g. Grimm et al., 2008; Lacoste et al., 2014) use standard depth intervals for prediction, based on national mapping requirements or suitable for their specific case study.

\subsection{Sampling design, sample size and density}

The sampling design is the spatial location of the sampling units used to calibrate or validate the ML algorithm. Most studies do not specify the sampling design used to generate the observations. It is speculated that the sample originates from multiple sources, e.g. legacy data, expert-based designs, and combination of several surveys, each of which had a different sampling design. When specified, nonprobability sampling such as grid-based sampling designs are by far the most used (e.g. by Pahlavan-Rad \& Akbarimoghaddam, 2018; Sergeev et al., 2019; Sharififar et al., 2019). Another non-probability sampling design is conditioned Latin Hypercube (cLHS), used to collect a sample in Lacoste et al. (2014); Brungard et al. (2015). Probability sampling is used in about one fourth of the studies. For example, simple random sampling is used in Tziachris et al. (2019), while a sample is collected based on stratified random sampling in Wiesmeier et al. (2011) using land use and topog- 
raphy as stratifying variables.

In our literature review, we found that the sample size varies considerably between studies. While the average sample is composed of 1,000 units, about one third of the studies use a sample with less than 150 units, mostly for local or small-scale regional areas. For example, Blanco et al. (2018) use a sample of size 47 for mapping soil water retention in a $93 \mathrm{~km}^{2}$ area while Massawe et al. (2018) observed 33 soil profiles to calibrate a ML algorithm and to predict soil taxa over a $11,600 \mathrm{~km}^{2}$ area. As expected, global studies have very large sample sizes. Hengl et al. (2017a) and Ramcharan et al. (2018) use a sample composed of more than 150,000 units to make soil property or class maps of the whole world, or of the United States, respectively.

When the sample size is associated to the extent of the study area, our review shows that large-scale studies have a very coarse sampling density. While the average sampling density in our literature is 0.24 units $/ \mathrm{km}^{2}$, studies by Beguin et al. (2017) and Wang et al. (2017) have both a sampling density smaller than 3 units $/ 10,000 \mathrm{~km}^{2}$ for mapping soil properties in the rangelands of eastern Australia or in the Canadian boreal forests. Small-scale studies have, conversely, high sampling density. All studies with area size less than $50 \mathrm{~km}^{2}$ have a sampling density larger than 7 units $/ \mathrm{km}^{2}$.

\subsection{What is mapped?}

\subsubsection{Quantitative variables}

ML algorithms have been successfully applied for quantitative mapping of various soil properties such as soil organic carbon concentration (Henderson et al., 2005; Bui et al., 2009; Kheir et al., 2010b; Dai et al., 2014; Siewert, 2018; Pouladi et al., 2019) and associated stocks (Grimm et al., 2008; Adhikari et al., 2014; Ließ et al., 2016; Wang et al., 2017; McNicol et al., 2019), to map soil texture (viz. clay, silt and sand content) (Ließ et al., 2012; Akpa et al., 2014; Vaysse \& Lagacherie, 2015; da Silva Chagas et al., 2016), pH (Dharumarajan et al., 2017), or cation exchange capacity (Forkuor et al., 2017).

ML algorithms have also been applied to make maps of soil nutrients such as nitrogen (Viscarra-Rossel et al., 2015; Forkuor et al., 2017), phosphorus (ViscarraRossel et al., 2015; Hengl et al., 2017b; Song et al., 2018), potassium, calcium or magnesium (Hengl et al., 2017b).

A number of studies have also predicted soil attributes and conditions with machine learning such as bulk density (Viscarra-Rossel et al., 2015) or soil pollutants 
(Kheir et al., 2010a). Wu et al. (2016) map soil background concentrations of arsenic in the Jiangxi Province in China. Taghizadeh-Mehrjardi et al. (2016) map soil salinity in Iran. Tajik et al. (2019) map soil invertebrate using environmental covariates in a deciduous forest ecosystem in northern Iran while Malone et al. (2009) map carbon storage and available water capacity in an area in eastern Australia.

\subsubsection{Categorical variables}

Compared with continuous soil property mapping, fewer studies apply ML to categorical variables. Digital mapping of soil classes using machine learning started in the 90s. Probably the first of its kind, Lagacherie \& Holmes (1997) predict soil classes in a regional area while Cialella et al. (1997) predict soil drainage classes using remote sensing and elevation covariates. Behrens et al. (2005) map soil units in a $600 \mathrm{~km}^{2}$ area of Western Germany. These studies have recently been completed by a number of publications comparing the maps predicted by a ML model to conventional soil maps (e.g. Zeraatpisheh et al., 2017). Scull et al. (2005); Brungard et al. (2015); Heung et al. (2016); Hounkpatin et al. (2018) employ machine learning to classify soil taxonomic units. Vermeulen \& Van Niekerk (2017) map salt-affected areas in irrigation schemes in South Africa. Table 1 provides an additional summary of case studies.

A special case of categorical mapping occurs when the map of soil class already exists but needs to be disaggregated. Bui et al. (1999) and Moran \& Bui (2002) use a decision tree to disaggregate an existing map and obtain a realization of the disaggregated soil class distribution. With multiple realizations, the most probable soil class is obtained for a given location. This is further investigated by Hansen et al. (2009) to disaggregate a reconnaissance soil map using a binary decision tree. A similar approach with decision tree is used in Häring et al. (2012) to downscale soil types within existing map unit boundaries. More recently, Odgers et al. (2014) use ML to model and disaggregate soil classes and report the probability associated to each soil class at a given location in the area of interest. A growing number of publication exploits the DSMART approach proposed by Odgers et al. (2014) (e.g. Holmes et al., 2014; Vincent et al., 2018; Ellili et al., 2019).

\subsection{Covariates}

Environmental covariates are used as predictors in ML algorithms. They are supposed to explain part of the physical and chemical process governing soil spatial variation. Most studies use about 20 covariates. Only a few use less than five (e.g. Dai et al., 2014; Padarian et al., 2019) while other use more than 100 (e.g Hengl 
et al., 2017a; Ramcharan et al., 2018). Since the covariates represent soil forming factors, numerous studies (e.g. Viscarra-Rossel \& Chen, 2011; Wang et al., 2018; Gomes et al., 2019; Szatmári \& Pásztor, 2019) logically select the covariates to represent the key factors of the scorpan model of soil spatial variation. The most common ones are existing soil property or class maps, (long-term) average annual precipitation and temperature, remote sensing images (e.g. SPOT satellite images or vegetation indices derived from satellite images), elevation, terrain attributes (e.g. slope, local curvature, topographic wetness index) and existing geological maps.

Covariates representing scorpan factor of soil variation might not be available or easily obtainable in all case studies. In some cases, covariates are chosen based on expert knowledge. A number of studies therefore calibrate machine learning algorithms using sets of climatic variables, remote sensing images or terrain attributes only, or a combination of them. For example, Mansuy et al. (2014) use a set of eight climatic and eight terrain attribute variables to map $\mathrm{C}, \mathrm{N}$ and soil texture in a large area in Canada. Sharififar et al. (2019) use six terrain attributes as predictors. There are chosen from a large set of environmental covariates using knowledge on the expected relationship between the covariate and the soil property to be mapped. We note that a few studies (e.g. Hengl et al., 2018; Miller et al., 2015a) consider that if a sufficiently large $(>100)$ number of covariates is used for calibration, the machine learning algorithm learns a representation of the spatial pattern and predicts a realistic spatial pattern. This large amount of covariates relies mostly on remote sensing images, e.g. MODIS land products (long-term averages, several nearor mid-infrared bands) or Landsat products (near-, short-wave near-infrared, or $\gamma$ radiometric bands, bare ground images).

A few studies account for the multi-scale variation of the environmental covariates. In other words, terrain derivatives may well be aggregated to account for physical processes in soil that are not visible are finer scale. Examples of studies using multi-scale covariates for mapping with machine learning algorithms are Behrens et al. (2010), Miller et al. (2015b) or more recently Behrens et al. (2018a). Miller et al. (2015b), for example, use a total of 412 covariates, several of which are derived from the aggregation of terrain attributes from a fine (i.e. a grid cell size of $2 \mathrm{~m} \times$ $2 \mathrm{~m}$ ) elevation map.

A growing number of studies have advocated the use of spatial surrogate covariates as an indicator of spatial position in the scorpan model of soil variation The most common surrogate is the use of geographical coordinates (easting and northing) 
as covariates in the model. Maps of distances from observation locations, or group of locations, have been used by Hengl et al. (2018). They are categorized into Euclidean, downslopes or "resistance" distances. More recently, Behrens et al. (2018b) use Euclidean distance fields, which are maps of distance from reference locations in the study area such as a corner or a center.

\subsection{Covariate selection}

Covariate ( $a k a$ feature) selection aims at reducing the number of covariates used to calibrate the machine learning models. While most ML models are robust to multicolinearity between covariates, there are several reasons for selecting a subset of covariates to calibrate the model. Some of them are: (i) to calibrate the ML model faster, (ii) to reduce complexity, (ii) to increase the prediction accuracy or (iv) to prevent over-fitting of the ML model, i.e. to prevent poor prediction accuracy on unseen data. In our literature review, about one third of the studies apply covariate selection. Two main categories of covariate selection techniques are found. The first applies the covariate selection as a pre-processing step, i.e. before calibrating the ML model. This is the case in Zhu et al. (2019); Hamzehpour et al. (2019); Zeraatpisheh et al. (2019). Hamzehpour et al. (2019) select the covariates to be used in calibration by computing the Pearson's $r$ correlation coefficient between the covariates, and by discarding the ones that were highly correlated, while Mosleh et al. (2016) select the covariates based on the Pearson $r$ correlation coefficient between the soil property values and the covariates, and select a subset of covariates which are strongly correlated with the property. The second type of covariate selection are called "wrapper" methods and rely on the inference made by a calibrated ML model to determine whether covariates are important. By re-calibrating a ML model several times, each time removing the least important covariate, one may expect to reduce considerably the overall number of covariates with little or no decrease in model prediction accuracy. Examples on the use of "wrapper" methods are found in Taghizadeh-mehrjardi et al. (2016); Shi et al. (2018); Rudiyanto et al. (2018); Tajik et al. (2019) or Gomes et al. (2019). The most used of "wrapper" methods is an optimization algorithm called recursive feature elimination.

\subsection{Machine learning models}

A large number of ML algorithms and their variants have been used in the DSM literature. For quantitative mapping, tree-based algorithms are the most popular ones, the simplest version of which is the regression tree, used for example by Taghizadeh-Mehrjardi et al. (2016). Regression tree is known to be sensitive to the 
calibration sample. To solve this problem, the bagging (bootstrap and aggregating) procedure (Breiman, 2017) has been introduced in random forest (RF). Our literature review shows that RF is currently the most popular ML algorithm for regression purposes. Example of case studies using RF for mapping are Tziachris et al. (2019); Vaysse \& Lagacherie (2015); Forkuor et al. (2017); Dharumarajan et al. (2017); Liu et al. (2019). More recently, Vaysse \& Lagacherie (2017) introduced a variant of random forest, called quantile regression forest, as a method to map the uncertainty associated with the prediction of the soil property. Another tree-based method is cubist, employed in about 10\% of the reviewed literature (e.g. by Mulder et al., 2016; Viscarra-Rossel et al., 2015; Miller et al., 2015a). A few studies (less than five) use boosted regression tree (Yang et al., 2016; Beguin et al., 2017). In addition, a number of studies use neural networks (Lamichhane et al., 2019) algorithms (Aitkenhead \& Coull, 2016; Guevara et al., 2018), such as artificial neural networks (Dai et al., 2014). A relatively small number of studies use alternative algorithms such as support vector machines (Guevara et al., 2018), $k$-nearest neighbours (Mansuy et al., 2014) or generalized boosted regression (Tziachris et al., 2019; Gomes et al., 2019).

For classification purposes, tree-based algorithms are also the most popular ones. About $80 \%$ of the case studies used at least one tree-based algorithm such as regression tree (e.g. Taghizadeh-Mehrjardi et al., 2019b; Heung et al., 2016), random forest (e.g. Häring et al., 2012) or boosted regression tree (e.g. Lorenzetti et al., 2015). Alternatively, gradient boosting is used by Hengl et al. (2017a), $k$-nearest neighbors by Vermeulen \& Van Niekerk (2017) and compared to support vector machines. The latter algorithm is also used in Taghizadeh-Mehrjardi et al. (2019b). Neural networks is also popular and used in Behrens et al. (2005); Heung et al. (2016).

Recent studies have proposed to use model ensemble techniques to improve the predicted map of several individual models in terms of accuracy. Taghizadeh-Mehrjardi et al. (2019a) combined seven ML model predictions for soil class mapping in a case study in Iran while Song et al. (2020) implemented a weighted ensemble learning model to map soil organic carbon in consideration of pedoclimatic zones in China. Ensembles are also considered in Hengl et al. (2017a) for global soil mapping.

\subsection{Parameter tunning}

The performance of a machine learning model is impacted by the values of its model parameters. While most ML would perform well on default tuning parameter values, almost half of the studies perform a search to find optimal values. Padarian et al. (2019) manually decide the artificial neural network neurons number for 
each layer of the network. This manual search is automated by a so-called gridsearch process. This is by far the most used technique for parameter tuning. In a grid-search process, a number of parameter values are evaluated based on the model prediction error. The process is computationally intensive (the ML model must be calibrated for each parameter set proposal). Examples of studies using a grid-search to find ML parameter values are Ottoy et al. (2017); Taghizadeh-Mehrjardi et al. (2016); Pahlavan-Rad \& Akbarimoghaddam (2018); Sergeev et al. (2019); Forkuor et al. (2017); Ramcharan et al. (2018). An alternative to the grid search is to apply an optimization algorithm, such as the particle swarm method, to find optimal parameter values. For example, Wu et al. (2016) compare two genetic algorithms and a grid search process to find the ML parameters. Recently, Wadoux et al. (2019b) use Bayesian optimization to optimize the number of layers, the neuron number, the learning rate and the batch size of an artificial neural network for mapping soil organic carbon.

\subsection{Validation and uncertainty quantification}

In our literature review, all studies compute at least one validation statistic to assess the quality of the prediction. A list of validation statistics is provided in Table 1. About $30 \%$ of the studies obtain the validation statistics through crossvalidation, while 30\% through data-splitting. The remaining studies either repeat data-splitting several times, validate through visual examination or use a grid-based sampling design. Only two studies collect an additional probability sample for validation (Subburayalu \& Slater, 2013; Lacoste et al., 2014).

In addition to the validation statistics, about $30 \%$ of the studies quantify the uncertainty associated with the prediction. These studies report confidence interval, obtained by bootstrapping the original set of observations (e.g. Chen et al., 2019; Padarian et al., 2019; Hamzehpour et al., 2019). A few studies use the kriging variance computed on the residuals of a trend obtained by predicting with a ML algorithm (e.g. Koch et al., 2019), or a combination of bootstrap and kriging variance (e.g. Viscarra-Rossel et al., 2015). In three studies, prediction intervals are obtained through the quantile regression forest. Wadoux (2019b) obtain the prediction intervals following a two-step procedure called mean plus variance estimate for mapping several soil properties using an artificial neural network. 
Table 1: Non-exhaustive list with summary of case studies in which machine learning algorithms are used for digital soil mapping.

\begin{tabular}{|c|c|c|c|c|c|c|c|c|c|}
\hline $\begin{array}{l}\text { Spatial } \\
\text { extent }^{1}\end{array}$ & Sample size & Sampling design & $\begin{array}{l}\text { Number of } \\
\text { covariates }\end{array}$ & $\begin{array}{l}\text { Machine learning } \\
\text { model }^{2}\end{array}$ & $\begin{array}{l}\text { Covariate } \\
\text { selection }\end{array}$ & $\begin{array}{l}\text { Parameter } \\
\text { tuning }\end{array}$ & $\begin{array}{l}\text { Validation statis- } \\
\text { tics }^{3}\end{array}$ & $\begin{array}{l}\text { Uncertainty } \\
\text { quantification }\end{array}$ & Reference \\
\hline \multicolumn{10}{|c|}{ Quantitative maps } \\
\hline Plot & 285 & grid-based & 19 & cubist, $\mathrm{RF}$ & no & no & $\mathrm{R}^{2}, \mathrm{RMSE}$ & no & Pouladi et al. (2019) \\
\hline Local & 47 & stratified random & 41 & $\mathrm{RF}$ & yes & yes & RMSE, IQR & yes & Blanco et al. (2018) \\
\hline Local & 70 & cLHS & 19 & cubist & no & no & $\begin{array}{l}\text { MAE, RMSE, } \mathrm{R}^{2} \text {, } \\
\text { CCC }\end{array}$ & yes & Lacoste et al. (2014) \\
\hline Local & 75 & grid-based & 9 & ANN & no & no & $\mathrm{R}^{2}, \mathrm{MSE}$ & no & Kalambukattu et al. (2018) \\
\hline Local & 98 & varied sources & 173 & $\mathrm{RF}$ & yes & no & RMSE, $\mathrm{R}^{2}$ & no & Shi et al. (2018) \\
\hline Local & 116 & simple random & 20 & $\mathrm{RF}$ & no & no & $\mathrm{R}^{2}, \mathrm{RMSE}, \mathrm{CCC}$ & no & Dharumarajan et al. (2017) \\
\hline Local & 117 & not specified & 412 & cubist & yes & no & $\begin{array}{l}\mathrm{ME}, \mathrm{MAE}, \mathrm{R}^{2} \\
\mathrm{R}_{a d j}^{2}\end{array}$ & no & Miller et al. (2015b) \\
\hline Local & 120 & stratified random & $\begin{array}{l}\text { not speci- } \\
\text { fied }\end{array}$ & $\mathrm{RF}$ & no & no & $\begin{array}{l}\mathrm{ME}, \mathrm{RMSE}, \mathrm{R}^{2} \text {, } \\
\mathrm{MSE}\end{array}$ & no & Wiesmeier et al. (2011) \\
\hline Local & 120 & stratified random & 22 & ANN, BRT & yes & yes & $\mathrm{R}^{2}, \mathrm{RMSE}, \mathrm{ME}$ & no & Mosleh et al. (2016) \\
\hline Local & 137 & systematic random & 20 & ANN, GEP & yes & yes & RMSE, $\mathrm{R}^{2}, \mathrm{MBE}$ & no & Mahmoudabadi et al. (2017) \\
\hline Local & 138 & not specified & 15 & $\mathrm{RF}$ & yes & no & RMSE, $\mathrm{R}^{2}, \mathrm{CCC}$ & no & Zhu et al. (2019) \\
\hline Local & 150 & grid-based & $\begin{array}{l}\text { not speci- } \\
\text { fied }\end{array}$ & ANN & no & yes & $\begin{array}{l}\text { correlation coeffi- } \\
\text { cient, } \mathrm{R}^{2}, \mathrm{RMSE}, \\
\text { Willmott's in- } \\
\text { dex of agreement, } \\
\text { RPIQ }\end{array}$ & no & Sergeev et al. (2019) \\
\hline Local & 151 & not specified & $\begin{array}{l}\text { not speci- } \\
\text { fied }\end{array}$ & & no & no & $\mathrm{R}^{2}, \mathrm{NRMSD}$ & no & Kovačević et al. (2010) \\
\hline Local & 153 & grid-based & 26 & $\mathrm{RF}$ & yes & no & RMSE, $\mathrm{R}^{2}$ & no & Tajik et al. (2019) \\
\hline Local & $159 / 34$ & not specified & 37 & $\begin{array}{l}\text { RF, cubist, QRF, NN, } \\
\text { avNNet, ctree, evtree, } \\
\text { GBM, } k \text {-NN, RT, } \\
\text { SVM }\end{array}$ & yes & no & $\begin{array}{l}\mathrm{R}^{2}, \text { RMSE, MAE, } \\
\text { MARE }\end{array}$ & yes & Rudiyanto et al. (2018) \\
\hline Local & 165 & stratified random & 18 & $\mathrm{RF}$ & no & yes & MSE, NMSE & no & Grimm et al. (2008) \\
\hline Local & 173 profiles & cLHS & 19 & $\mathrm{Rf}$ & no & no & ME, RMSE, $\mathrm{R}^{2}$ & no & $\begin{array}{l}\text { Taghizadeh-Mehrjardi et al. } \\
\text { (2014) }\end{array}$ \\
\hline Local & 188 profiles & cLHS & 16 & $\begin{array}{l}\mathrm{ANN}, \mathrm{SVR}, k-\mathrm{NN}, \\
\mathrm{RF}, \mathrm{RT}\end{array}$ & no & yes & RMSE, CCC & no & $\begin{array}{l}\text { Taghizadeh-Mehrjardi et al. } \\
(2016)\end{array}$ \\
\hline Local & 234 & not specified & 410 & cubist & yes & no & MAE, $\mathrm{R}^{2}$ & yes & Miller et al. (2015a) \\
\hline
\end{tabular}

\footnotetext{
${ }^{1}$ Plot: 0-1 km² ; Local: $>1 \mathrm{~km}^{2}-10^{4} \mathrm{~km}^{2}$; Regional: $>10^{4} \mathrm{~km}^{2}-10^{7} \mathrm{~km}^{2}$; Global: $>10^{7} \mathrm{~km}^{2}$.

${ }^{2} \mathrm{RF}$ : random forest; ANN: artificial neural networks; CNN: convolutional neural networks; GBM: gradient boosting machine; BRT: boosted regression tree; GEP: gene expression programming; QRF: quantile regression forest; avNNet: neural networks using model averaging; ctree: conditional inference trees; evtree: evolutionary algorithm for classification and regression tree; NN: neural networks; GBM: generalized boosted regression; $k$-NN: $k$-nearest neighbors; RT: regression tree; SVM: support vector machine; MARS: multivariate adaptive regression splines; SGB: stochastic gradient boosting; CART: classification and regression tree; NSC: nearest shrunken centroids; CT: classification tree; BCT: bagged classification tree; DT: decision tree; LMT: logistic model tree; EGB: extreme gradient boosting.

${ }^{3} \mathrm{R}^{2}$ : coefficient of determination; $\mathrm{R}_{a d j}^{2}$ : adjusted coefficient of determination; RMSE: root mean square error; IQR: interquartile range; MAE: mean absolute error; CCC: Lin's concordance correlation coefficient; MSE: mean square error; ME: mean error; MBE: mean bias error; RPIQ: ratio of performance to interquartile distance; NRMSD; normalized root mean squared deviation; MARE: median absolute relative error; NMSE: normalized mean square error; sMAPE: symmetric mean absolute percentage error; SS: skill score; RMSD: minimum root mean square deviation; RPD: residual prediction deviation; SDE: standard deviation of the error; EC: overall ratio; OA: overall accuracy; PA: producer accuracy; UA; user accuracy; AUROC: area under receiver operating characteristic curve; AUC: area under the curve.
} 


\begin{tabular}{|c|c|c|c|c|c|c|c|c|c|}
\hline Local & 330 profiles & not specified & 12 & $\begin{array}{l}\text { BRT, ANN, least- } \\
\text { square SVM }\end{array}$ & no & yes & $\begin{array}{l}\mathrm{R}^{2}, \mathrm{R}_{a d j}^{2}, \mathrm{RMSE}, \\
\text { relative RMSE }\end{array}$ & no & Ottoy et al. (2017) \\
\hline Local & 330 & simple random & 10 & $\mathrm{RF}, \mathrm{GBM}$ & no & yes & $\begin{array}{l}\text { ME, MAE, RMSE, } \\
\mathrm{R}^{2}\end{array}$ & & Tziachris et al. (2019) \\
\hline Local & 334 & cLHS & 16 & cubist, RF, RT & yes & no & $\mathrm{R}^{2}, \mathrm{RMSE}$ & no & Zeraatpisheh et al. (2019) \\
\hline Local & $342 / 321$ & - & 14 & $\begin{array}{l}\text { MARS, SVR, RF, } \\
\text { Cubist, NN }\end{array}$ & - & yes & $\mathrm{R}^{2}$ & no & Behrens et al. (2018b) \\
\hline Local & 399 & not specified & 12 & $\mathrm{RF}$ & no & no & $\mathrm{R}^{2}, \mathrm{RMSE}$ & no & da Silva Chagas et al. (2016) \\
\hline Local & 440 & varied sources & 19 & RF, SVM, ANN & no & yes & RMSE, ME & no & Were et al. (2015) \\
\hline Local & 460 & grid-based & 21 & $\mathrm{RF}$ & no & yes & ME, MAE, RMSE & no & $\begin{array}{l}\text { Pahlavan-Rad \& Akbarimoghad- } \\
\text { dam (2018) }\end{array}$ \\
\hline Local & 568 & simple random & 26 & QRF & no & no & $\begin{array}{l}\mathrm{R}^{2}, \text { RMSE, range- } \\
\text { nomalized RMSE, } \\
\text { Moran's I }\end{array}$ & yes & Kirkwood et al. (2016) \\
\hline Local & 1104 & expert & 29 & RF, SVM, SGB & no & yes & RMSE, sMAPE & no & Forkuor et al. (2017) \\
\hline Local & $\begin{array}{l}\leq 1052 / 2050 / \\
2379\end{array}$ & varied sources & $300-500$ & BRT, RF & yes & yes & $\begin{array}{l}\text { bias, RMSE, SS, } \\
\mathrm{R}^{2}\end{array}$ & no & Nussbaum et al. (2018) \\
\hline Local & 2388 & varied sources & 3 & CNN, RF & no & yes & $\begin{array}{l}\text { ME, RMSE, } \mathrm{R}^{2}, \\
\text { CCC }\end{array}$ & no & Wadoux et al. (2019b) \\
\hline Regional & not specified & not specified & 20 & cubist & no & no & $\begin{array}{l}\mathrm{R}^{2}, \mathrm{RMSE}, \text { bias, } \\
\mathrm{CCC}\end{array}$ & yes & Mulder et al. (2016) \\
\hline Regional & 125 profiles & purposive & 12 & BRT, RF & no & no & $\begin{array}{l}\text { MAE, RMSE, } \mathrm{R}^{2} \text {, } \\
\text { CCC }\end{array}$ & no & Yang et al. (2016) \\
\hline Regional & 244 & grid-based & 4 & ANN & no & yes & $\begin{array}{l}\text { ME, MAE, RMSE, } \\
\text { CCC }\end{array}$ & no & Dai et al. (2014) \\
\hline Regional & $339 / 961$ & varied sources & 40 & QRF & no & no & $\mathrm{R}^{2}, \mathrm{RMSE}$ & yes & Nauman \& Duniway (2019) \\
\hline Regional & 485 profiles & not specified & 5 & CNN & no & yes & $\mathrm{R}^{2}, \mathrm{RMSE}$ & yes & Padarian et al. (2019) \\
\hline Regional & 500 & not specified & 12 & $\mathrm{RF}, \mathrm{BRT}$ & yes & no & $\mathrm{R}^{2}, \mathrm{RMSE}$ & no & Beguin et al. (2017) \\
\hline Regional & 528 & $\begin{array}{l}\text { subset from a } \\
\text { systematic grid }\end{array}$ & 18 & $k$-NN & yes & no & $\begin{array}{l}\text { RMSE, } R^{2} \text {, Bias, } \\
\text { coefficient of vari- } \\
\text { ance }\end{array}$ & no & Mansuy et al. (2014) \\
\hline Regional & 705 & simple random & 16 & RF, BRT, SVM & yes & yes & $\begin{array}{l}\mathrm{R}^{2}, \mathrm{MAE}, \mathrm{RMSE}, \\
\mathrm{CCC}\end{array}$ & yes & Wang et al. (2018) \\
\hline Regional & 978 profiles & not specified & 24 & $\mathrm{RF}$ & no & no & $\begin{array}{l}\mathrm{R}^{2}, \mathrm{ME}, \mathrm{RMSE} \\
\mathrm{CCC}\end{array}$ & no & Akpa et al. (2014) \\
\hline Regional & 1,014 & stratified random & 327 & $\begin{array}{l}\text { CART, BRT, BRT, } \\
\text { RF, SVM }\end{array}$ & yes & no & $\begin{array}{l}\mathrm{R}^{2}, \mathrm{RMSD}, \mathrm{RPD}, \\
\mathrm{RPIQ}\end{array}$ & no & Keskin et al. (2019) \\
\hline Regional & 1,134 & not specified & 81 & NN & no & no & $\begin{array}{l}\mathrm{R}^{2}, \mathrm{ME}, \mathrm{MAE}, \\
\mathrm{RMSE}\end{array}$ & no & Aitkenhead \& Coull (2016) \\
\hline Regional & 1,300 profiles & not specified & 6 & $\mathrm{RF}$ & no & no & CCC, RMSE & yes & McNicol et al. (2019) \\
\hline Regional & 1,626 & not specified & 40 & SVM & no & yes & $\mathrm{R}^{2}, \mathrm{MSE}$ & no & Wu et al. (2016) \\
\hline Regional & 2,024 profiles & legacy data & 16 & QRF & no & no & $\begin{array}{l}\text { ME, RMSE, } \mathrm{R}^{2} \text {, } \\
\text { accuracy plot }\end{array}$ & yes & Vaysse \& Lagacherie (2017) \\
\hline Regional & 2,024 profiles & legacy data & 16 & & no & yes & MSE, $R^{2}$ & no & Vaysse \& Lagacherie (2015) \\
\hline Regional & 2,943 & $\begin{array}{l}\text { two-stage system- } \\
\text { atic }\end{array}$ & 37 & CNN, RF & no & yes & $\begin{array}{l}\text { ME, RMSE, } \mathrm{R}^{2}, \\
\mathrm{CCC}\end{array}$ & yes & Wadoux (2019b) \\
\hline Regional & 4,859 & not specified & 26 & QRF & no & no & $\begin{array}{l}\text { ME, RMSE, accu- } \\
\text { racy plot }\end{array}$ & yes & Szatmári et al. (2019) \\
\hline Regional & 4,859 & not specified & 32 & QRF & no & no & $\begin{array}{l}\text { ME, RMSE, accu- } \\
\text { racy plot }\end{array}$ & yes & Szatmári \& Pásztor (2019) \\
\hline Regional & 5,386 & varied sources & 6 & cubist, SVM & no & no & $\mathrm{R}^{2}, \mathrm{MSE}, \mathrm{CCC}$ & & Somarathna et al. (2016) \\
\hline Regional & 13,000 & not specified & 18 & $\mathrm{RF}$ & no & no & $\mathrm{R}^{2}$ & yes & Koch et al. (2019) \\
\hline Regional & 19,790 & $\begin{array}{l}\text { two-stage system- } \\
\text { atic }\end{array}$ & 197 & $\mathrm{RF}$ & no & no & $\mathrm{ME}$ & no & Wadoux et al. (2019a) \\
\hline Regional & 37,693 & legacy soil data & 74 & RF, Cubist, SVM & yes & yes & $\mathrm{R}^{2}, \mathrm{RMSE}, \mathrm{MAE}$ & yes & Gomes et al. (2019) \\
\hline $\begin{array}{l}\text { Regional } \\
\text { - Global }\end{array}$ & $2,268-27,262$ & varied sources & 34 & cubist & no & yes & $\begin{array}{l}\text { CCC, RMSE, } \\
\text { SDE, ME }\end{array}$ & yes & Viscarra-Rossel et al. (2015) \\
\hline
\end{tabular}




\begin{tabular}{|c|c|c|c|c|c|c|c|c|c|}
\hline $\begin{array}{l}\text { Regional } \\
\text { - Global }\end{array}$ & 366,034 & varied sources & $>200$ & $\mathrm{RF}, \mathrm{GBM}$ & no & yes & $\begin{array}{l}\mathrm{R}^{2}, \mathrm{ME}, \mathrm{RMSE} \\
\mathrm{MAE}\end{array}$ & yes & Ramcharan et al. (2018) \\
\hline Global & 11,268 & legacy soil data & 118 & $\begin{array}{l}\text { SVM, kernel weighted } \\
\text { NN, RF }\end{array}$ & yes & no & $\mathrm{EC}, \mathrm{RMSE}, \mathrm{R}^{2}$ & yes & Guevara et al. (2018) \\
\hline Global & 150,000 & legacy soil data & $>200$ & $\mathrm{RF}, \mathrm{GBM}$ & no & yes & $\mathrm{R}^{2}$ & no & Hengl et al. (2017a) \\
\hline \multicolumn{10}{|c|}{ Categorical maps } \\
\hline Local & - & not specified & 125 & ANN & no & no & $\begin{array}{l}\text { Accuracy, recall, } \\
\text { precision }\end{array}$ & no & Behrens et al. (2005) \\
\hline Local & 33 profiles & not specified & 16 & RF, J48 & no & no & not specified & no & Massawe et al. (2018) \\
\hline Local & $103 / 297 / 57$ & cLHS & 130 & $\begin{array}{l}k \text {-NN, NSC, CT, } \\
\text { BCT, RF, linear SVM, } \\
\text { radial-basis SVM, NN, } \\
\text { ANN }\end{array}$ & yes & yes & $\begin{array}{l}\text { Kappa analysis, } \\
\text { Brier scores, vi- } \\
\text { sual inspection, } \\
\text { confusion index }\end{array}$ & no & Brungard et al. (2015) \\
\hline Local & 125 profiles & cLHS & 17 & $\mathrm{RF}$ & no & no & $\begin{array}{l}\text { map purity, Co- } \\
\text { hen's kappa, Shan- } \\
\text { non entropy index, } \\
\text { relative purity, } \\
\text { relative diversity }\end{array}$ & no & Zeraatpisheh et al. (2017) \\
\hline Local & 151 & not specified & $\begin{array}{l}\text { not speci- } \\
\text { fied }\end{array}$ & SVM & no & no & $\begin{array}{l}\text { NRMSD, mi- } \\
\text { cro averaged F1 } \\
\text { measure, kappa } \\
\text { statistics }\end{array}$ & no & Kovačević et al. (2010) \\
\hline Local & 175, 63 profiles & varied sources & 27 & $k$-NN, SVm, DT, RF & no & no & $\begin{array}{l}\text { OA, PA, UA, } \\
\text { kappa coefficient, } \\
\text { AUROC }\end{array}$ & no & Vermeulen \& Van Niekerk (2017) \\
\hline Local & 452 profiles & regular grid & 6 & $\mathrm{DT}, \mathrm{RF}$ & yes & no & $\begin{array}{l}\text { OA, UA, PA, } \\
\text { Kappa coefficient } \\
\text { of agreement }\end{array}$ & no & Sharififar et al. (2019) \\
\hline Local & 917 & grid-based & 33 & RF & yes & no & Kappa index & no & Hounkpatin et al. (2018) \\
\hline Local & 3,121 & $\begin{array}{l}\text { by-polygon, } \\
\text { equal-class, area- } \\
\text { weighted, and } \\
\text { area-weighted } \\
\text { with random over } \\
\text { sampling }\end{array}$ & 20 & $\begin{array}{l}\text { CART, CART with } \\
\text { bagging, RF, } k \text {-NN, } \\
\text { NSC, ANN, LMT, } \\
\text { SVM }\end{array}$ & no & yes & $\begin{array}{l}\text { overall agreement, } \\
\text { quantity disagree- } \\
\text { ment, allocation } \\
\text { disagreement, total } \\
\text { disagreement }\end{array}$ & no & Heung et al. (2016) \\
\hline Regional & 89,323 & random sampling & 26 & $k$-NN, RF & yes & no & recall, accuracy & no & Subburayalu \& Slater (2013) \\
\hline Regional & 366,034 & varied sources & $>200$ & RF, GBM & no & yes & $\begin{array}{l}\text { OA, regional } \\
\text { dataset }\end{array}$ & yes & Ramcharan et al. (2018) \\
\hline Regional & 7,664 profiles & varied sources & 110 & $\begin{array}{l}\text { DT, RF, EGB, SVM, } \\
k \text {-NN }\end{array}$ & yes & no & $\begin{array}{l}\text { OA, precision, } \\
\text { recall, F-score, } \\
\text { K-index }\end{array}$ & no & $\begin{array}{l}\text { Taghizadeh-Mehrjardi et al. } \\
\text { (2019b) }\end{array}$ \\
\hline Regional & 9,924 & not specified & 23 & $\mathrm{RF}$ & yes & no & error matrix & no & Häring et al. (2012) \\
\hline Global & 150,000 & legacy data & $>200$ & RF, GBM & no & yes & $\begin{array}{l}\text { map purity, } \\
\text { weighted kappa } \\
\text { metrics, AUC, } \\
\text { True positive rate, } \\
\text { scaled Shannon's } \\
\text { entropy index }\end{array}$ & no & Hengl et al. (2017a) \\
\hline
\end{tabular}




\section{Challenges and opportunities}

Based on the review, here we identify some knowledge gaps and challenges in the current use of ML algorithms for DSM. We will outline some opportunities for research.

\subsection{Sampling}

Despite abundant evidence that the sampling design and sample size play a key role in the resulting map accuracy (De Gruijter et al., 2006), sampling designs suitable for mapping with machine learning are yet to be uncovered. The impact of the sample size for mapping with ML is discussed in Somarathna et al. (2017) where the efficiency of several ML algorithms are compared for the spatial prediction of soil carbon. The study shows that having a sufficiently large sample size is more important than choosing a sophisticated ML algorithm, and that when the sample size is small, it it best to use simple models. About sampling designs, Brus (2019) speculates that machine learning algorithms would benefit from a spread of the sampling units in the feature (covariate) space, and suggests the use of feature space coverage sampling (FSCS) using $k$-means clustering or conditioned Latin Hypercube sampling (cLHS). Both sampling designs aim at covering the space spanned by the covariates, but in different ways. Experimental results are provided by Wadoux et al. (2019a) in a study comparing five sampling designs (viz. simple random sampling, cLHS, spatial coverage sampling (SCS), FSCS and a design optimized in terms of mean square error) for soil property mapping with random forest. The results show large differences in mapping accuracy between the designs, and that a FSCS design optimized in the most important covariates of the random forest model had the closest match to an optimized design. By performing further diagnostics, the study concludes that RF does not benefit from a uniform spread of the units in the geographic/feature space, nor from reproducing the marginal distribution of the covariates (as it is done in cLHS). These results apply for RF but there is a need to further investigate sampling designs for other machine learning algorithms. While most studies in our literature review (Table 1) use a grid-based sampling or cLHS, there is now evidence that most conventional sampling designs (e.g. spatial coverage sampling) are not effective for the purpose of mapping with machine learning.

To discover what makes a good design for mapping with machine learning, one should ideally derive optimal designs. More importantly, one should investigate the characteristics of these designs, so that future research can generate simple designs that resemble the optimal ones (Wadoux, 2019a). It is likely that optimal designs differ between machine learning algorithms. We speculate that a somewhat uniform 
spread in the feature (i.e. covariate) space remains important for all ML algorithms since they all link the covariates and the sample values in a non-linear way, but that additional considerations might outweigh or overtake this uniform spread. An example of optimal design is given by the studies of Pozdnoukhov \& Kanevski (2006) and Tuia et al. (2013) where the sampling configurations are optimized with active learning for mapping with support vector machines. In the first study, the selected sampling units are the most beneficial for the algorithm, avoiding mis-classification between temperature below or above 20Cs (categorical mapping) by becoming support vectors. In Tuia et al. (2013), a similar methodology is adopted and tested in three case studies to subsample an existing sample for quantitative mapping, to add optimally new sampling units in a continuous map or to define suitable areas for sampling. In all case studies, the authors obtained a design optimal for the purpose of mapping with support vectors machine. They conclude that while a sampling design can be representative of the geographical space, the latter can be judged unrepresentative if other dimensions are considered. These results encourage the use of new methods for sampling design optimization such as active learning. Active learning is a model-based sequential re-design algorithm. In active learning, the objective function (e.g. the spatially averaged prediction uncertainty) is explicitly quantified and used to define the additional sampling units that are the most beneficial for the model (e.g. the boundary between two classes). In this sense, active learning is similar to optimization with spatial simulated annealing routinely used in geostatistical sampling design optimization. Besides the optimization algorithms, a set of objective functions needs to be tested. MacKay (1992) defined an objective function that searches for the optimal units in the space spanned by the predictors (i.e. covariates) for prediction using a neural network algorithm. Taking the latter considerations and testing active learning for sampling design optimization would certainly make a valuable contribution to digital soil mapping research.

\subsection{Resampling}

Regional and global scale studies almost invariably use legacy soil data (Stumpf et al., 2016). Legacy soil samples provide valuable information on soil classes and properties but are often highly clustered in areas of specific interest. In modelling with machine learning, it is assumed that the sample is composed of independent and identically distributed sampling units whereas soil observations within an area typically exhibit spatial autocorrelation (i.e close observations are more similar than remote ones) This has important implications in terms of sampling, resampling of the observations and validation of the models. A ML algorithm calibrated with a spatially clustered sample may lead to biased predictions over the area because of 
the over-representation in the calibration process of regions of high sampling density. Despite being critical, this has yet been disregarded in DSM studies. In geostatistics, spatial declustering has been applied to reduce the effect of clustered data in the calculation of experimental variogram (Marchant et al., 2013). One form, called cell declustering involves overlaying a grid over the area and assigning a weight to the sampling units based on the inverse of the number of units in the cell. In ecology, a first attempt was made by Bel et al. (2005) and later Bel et al. (2009) to decluster the sampling units used in the calibration of a CART model. In Bel et al. (2005), weights are given to the sampling units, where the weights are obtained from a kriging of the spatial mean. Bel et al. (2009) elaborate a more complex procedure in which all quantities involved in the CART algorithm (e.g. the proportion of leaves) have a spatial estimate. This has been further considered by Stojanova et al. (2013) for both categorical and quantitative mapping of ecological variables. Illés et al. (2019) applied polygonal declustering technique to spatially clustered samples by assigning weights on the units based on Voronoi's area proportion.

We point out that the clustering may also occur in the feature (i.e. covariates) space and speculate that this may also affect the prediction if most units are clustered at some specific areas of the feature space. For example, a model trained to predict organic carbon in a mountainous area will exhibit biased prediction if most sampling units originate from valley, and that elevation is used as predictors. Similar to Bel et al. (2005), weights can be assigned to the units so down-weight the importance of over-sampled areas in the feature space. An example method is provided by Carré et al. (2007). The authors assume that a good sample have a uniform spread in the feature space and thus covers all strata of a hypercube based on the covariates. A weight is assigned to each unit in the sample based on the density of the units in each stratum. The larger the density within the stratum, the smaller the weight assigned to a single unit.

The nature of the legacy soil data in categorical mapping also poses additional challenges. ML algorithms for categorical mapping rely on balanced sets of units. In other words, all classes shall comprise a comparable number of sampling units. Legacy soil samples are considered imbalanced in that all classes are not represented equally. Most ML algorithms are calibrated by maximizing the average (classification) accuracy on an independent validation sample. This often results in very low predictive accuracy for under-sampled classes, and models biased toward the oversampled classes (He \& Garcia, 2009). In the ML literature, several approaches have been developed to handle class imbalanced samples. At the higher level, one may 
distinguish between cost function and resampling based approaches. In the first approach, the model is penalized for miss-classification to under-represented classes. This stems from the calibration of ML algorithms, which minimize a loss function to find optimal parameter values (e.g. in neural networks). In the second approach, resampling of the sample is performed by either adding units in the under-sampled class, removing units from the over-sampled class, or a mix of the two. The second approach has been recently been applied in soil mapping studies, in particular by Heung et al. (2016) and Sharififar et al. (2019). Taghizadeh-Mehrjardi et al. (2019b) tested eight resampling approaches and their effect on the prediction accuracy of five ML algorithms in two large-scale case studies. However, to date resampling techniques are applied the same way as in other disciplines while soil data often presents spatial autocorrelation which may impact the resampling strategies. This has not yet been investigated in the literature. The integration of resampling strategies within a general framework for mapping with ML is provided in Fig. 3.

\subsection{Accounting for spatial information}

Machine learning algorithms do not account for spatial autocorrelation contained in the raw soil data, unless explicitly specified. Sinha et al. (2019) have tested random forest for different scenarios of spatial autocorrelation in the observations and confirmed that the presence of spatial autocorrelation leads to high variance of the residuals. ML algorithms accounting for autocorrelated observations have recently been formulated, such as geographical random forest (Georganos et al., 2019), or spatial ensemble techniques (Jiang et al., 2017). The two methods boil down to geographically weighted regression by fitting spatially local sub-models using only neighbouring observations. Jiang et al. (2017) decomposed the area into geographic disjoint sub-areas, and fitted a local model in each sub-area. Georganos et al. (2019) fitted a sub-model to each observation using random forest, accounting for both nonstationarity and spatial autocorrelation.

Applying a non-spatial model for digital soil mapping is not a problem in itself. This is corroborated by the definition of DSM given in Lagacherie \& McBratney (2006), which gives provision for mapping using "non-spatial soil inference systems". In theory, if one includes all relevant environmental variables to model the soil property or class, there should be no spatial autocorrelation in the residuals of the fitted models. If this happens, some important predictors are likely to be missing. More importantly, this also means that predictions made by the ML algorithm might be biased or the model underfitted because this is a violation of the assumption of independence between data points that is implicitly assumed. Kühn \& Dormann (2012) 
recommend mapping the spatial distribution of the residual autocorrelation to facilitate the identification of a missing spatial process. In some cases, a map of residuals exhibits a clear pattern (e.g. increasing residuals with distance from the river) and might help to generate a new hypothesis or to refine the existing model (see Fig. 3).

Despite the availability of datasets and care made during modelling, residual autocorrelation is still likely to occur. Several authors have advocated the use of spatial surrogate covariates as an indicator of spatial position in the scorpan model of soil variation or to account for spatial autocorrelation contained in the data. The most common surrogate is the use of geographical coordinates (easting and northing) as covariate in the model. This has led to maps with visible artefacts, in particular when used in combination with tree-based algorithms. Alternatively, maps of distances from observation locations, or a group of locations, have been proposed by Hengl et al. (2018). They are categorized into Euclidean, downslopes or "resistance" distances. Maps of distance to observation locations generally have no direct meaning in terms of soil process over an area (e.g. distance from the river). Behrens et al. (2018b) propose to use Euclidean distance fields, which are maps of distance from reference locations in the study area such as the corner or the centre. The studies using distance maps as covariates have shown for several case studies an important reduction of the residual autocorrelation, when compared to a model without distance maps in the set of covariates.

In the context of digital soil mapping, we infer that the current use of distance maps is not satisfactory for several reasons. Including pseudo-covariates with the set of pedologically relevant covariates can be harmful because it precludes analysis of the residuals and the generation of new hypotheses from these residuals (Hawkins, 2012). It also hampers the interpretation of the most important predictors (Meyer et al., 2019), which is key in several studies on soil mapping. Finally, pseudo-covariates of distance may well integrate over several pedologically relevant covariates, making them better predictors or masking the effect of pedologically relevant covariates. In spatial ecology, alternatives to distance maps are found in the use of spatial eigenvector maps, spatial filters or trend-surface regression computed on, or optimized for, the residuals of a model calibrated using ecologically relevant covariates (Kühn et al., 2009). The process is generally in three steps (Fig. 1). In the first step, the variable of interest is fitted using ecologically relevant covariates, and the (autocorrelated) residuals are mapped to investigate whether there is an obvious missing spatial process in the model. In the second step, spatial surrogate covariates are computed on, or optimized for, the residuals. Finally, a model is calibrated using the covariates 
from steps 1 and 2 .

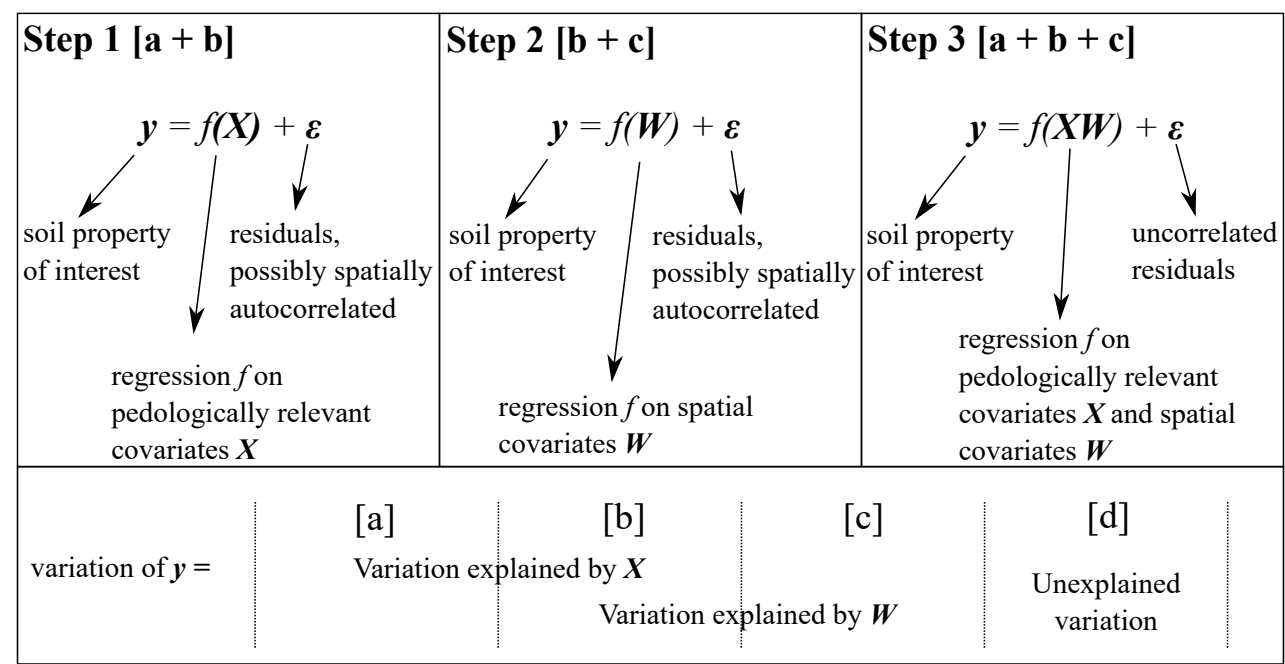

Figure 1: The three steps of variation partitioning between environmental $\mathbf{X}$ and spatial covariates $\mathbf{W}$. The variation of $\mathbf{y}$ is partitioned into four fractions (Peres-Neto et al., 2006) which are [a] the variation due to the environmental covariates, [b] the variation due to the spatial component of the environmental variables, $[\mathrm{c}]$ the spatial component and [d] the unexplained residual variation. Each component is estimated using the amount of variance explained. All $[a+b+c+d]$ sum to 1 .

The main advantage is to enable subsequent interpretation of the role of environmental covariates, spatial covariates (most often in the form of Moran's eigenvector maps) and unexplained (uncorrelated) residuals (the "ignorance") using variation partitioning techniques (Peres-Neto et al., 2006). Figure 1 shows that $[\mathrm{a}+\mathrm{b}]$ is the relative influence of environmental variables to the model prediction while $[\mathrm{b}+\mathrm{c}]$ is the relative influence of spatial covariates. The component [b] is the shared variation of $[\mathrm{a}]$ and $[\mathrm{c}]$ because environmental covariates are spatially structured. The remaining component $[\mathrm{d}]$ computed by $1-[\mathrm{a}+\mathrm{b}+\mathrm{c}]$ is the residual fraction of the variation. Another benefit of this approach is to have spatial surrogate covariates with little or no correlation with the meaningful environmental covariates. This approach has not yet been tested in DSM, but it would certainly make a valuable contribution to increase the interpretability of the ML models and their account of the spatial autocorrelation contained in soil data.

\subsection{Multivariate mapping}

Several authors (e.g. Hengl et al., 2018; Wadoux, 2019b; Wadoux et al., 2019b; Padarian et al., 2019) have shown that it is possible to calibrate a single ML model 
to predict either multiple soil properties or a single soil property at multiple depths. This reduces the risk of overfitting, computational resources that would be otherwise required to calibrate several disjoint models (Wadoux, 2019b), and increases prediction accuracy if there is correlation between the variables to predict. Padarian et al. (2019) use a multivariate CNN model to predict SOC at multiple soil depths and report a significant increase of prediction accuracy for the deeper soil depths, compared to predictions made for each depth separately by a cubist model. Wadoux (2019b) have shown that for a NN model, it was feasible to constrain the prediction to avoid inconsistent prediction between compositional soil properties, in particular soil texture. It was done by adding an additional layer to the model, but we speculate that this could also be realized by modifying the objective function used to calibrate the model. Despite a few recent studies, there has been little interest in multivariate soil mapping using ML algorithms. In the ML literature, it appears that almost all conventional ML algorithms have a multivariate counterpart. Multivariate NNs have already been tested in soil mapping studies. An adaptation of the RF algorithm for multivariate mapping is proposed by Hengl et al. (2018) but has several limitations. For example, the calibrated model size increases dramatically when the number of soil properties to predict also increases and it does not allow to separate the contribution of the covariates to each predicted property separately. A theoretical framework for multivariate RF is described by Segal \& Xiao (2011) and was further implemented in the R language by Rahman et al. (2017). For support vector machines, a multivariate extension is described in $\mathrm{Xu}$ et al. (2013).

One objective when mapping soil properties or classes is to learn from the calibrated model. A calibrated multivariate model can provide insights on the soil property and horizon interrelations. Regrettably, in a multivariate machine learning model, the correlation between soil properties or depths is not modelled explicitly (e.g. using a cross-covariance matrix between soil properties). As a result, the correlation between properties or depths cannot be assessed internally and no pedological interpretation can be derived from the calibrated model. More research is needed on whether the correlation between original and predicted soil properties (or depths) is preserved in a multivariate ML model. To model the correlation between properties explicitly, two solutions are possible. The first is to calibrate additional stochastic parameters together with the ML parameters (e.g. in a neural network algorithm). This can take the form of an auto-regressive model between the predictions (Uria et al., 2016). Another straightforward solution is to calibrate the model with a criterion related to the absolute difference in correlation between the measured properties and predicted properties. While this is easy to implement in ML calibration based 
on an objective function (e.g. neural network), this is not straightforward for models such as RF. Overall, including correlation between properties or depths when predicting with a ML algorithm requires further investigation so as to build pedologically realistic and interpretable models.

\subsection{Uncertainty analysis}

Uncertainty analysis in digital soil mapping is crucial to deciding whether the predicted soil map is reliable to be used for agricultural production systems or decision making. Uncertainty analysis is also about knowing better the limits of the models and is therefore one step towards model interpretability. At the higher level, the machine learning literature distinguishes two sources of uncertainty: aleatoric and epistemic uncertainties (Fig. 2). Aleatoric uncertainty is the data noise variance (in other terms, the data error), and arises from noise in the data and measurement error. Epistemic uncertainty refers to model and model parameter uncertainty and represents our ignorance about a true model that generated the data. While epistemic uncertainty is easy to reduce (e.g. by collecting more data at areas of low sampling density), aleatoric uncertainty is rather difficult to assess (one must repeat the measurement several times) and even more to reduce. Methods to quantify epistemic uncertainty are bootstrapping, or Bayesian modelling. Quantifying epistemic uncertainty enables to obtain confidence intervals of the prediction. Aleatoric uncertainty is mainly quantified by quantile regression methods, but Monte-Carlo simulation from the probability distribution of the observations might also be a possible approach. The quantification of both aleatoric and epistemic uncertainty provides prediction intervals with methods such as quantile regression forest (QRF), the Delta or Bayesian methods and the mean plus variance estimate (MVE) for neural network algorithms.

The recent development of conditional generative adversarial networks (cGAN) (Mirza \& Osindero, 2014) to generate possible realizations of the observations with specific conditions or characteristics seem to be of particular interest to include measurement error in DSM. Including measurement error is considered by Wadoux et al. (2019b) for mapping soil organic carbon using uncertain measurement of the soil property. However, the authors do not propose a method to quantify the uncertainty of the measurements, nor propagate the measurement error to the predicted map. With cGAN, a probability distribution of the observations is built, which might be used for Monte Carlo simulations. Each Monte Carlo sample is used as input in the ML algorithm, and the final map is the integration of all these simulations. This would effectively tackle the aleatoric uncertainty of the ML model. More impor- 
tantly, this would also quantify the uncertainty present in the measurements, which is currently one of the most important challenges in DSM.

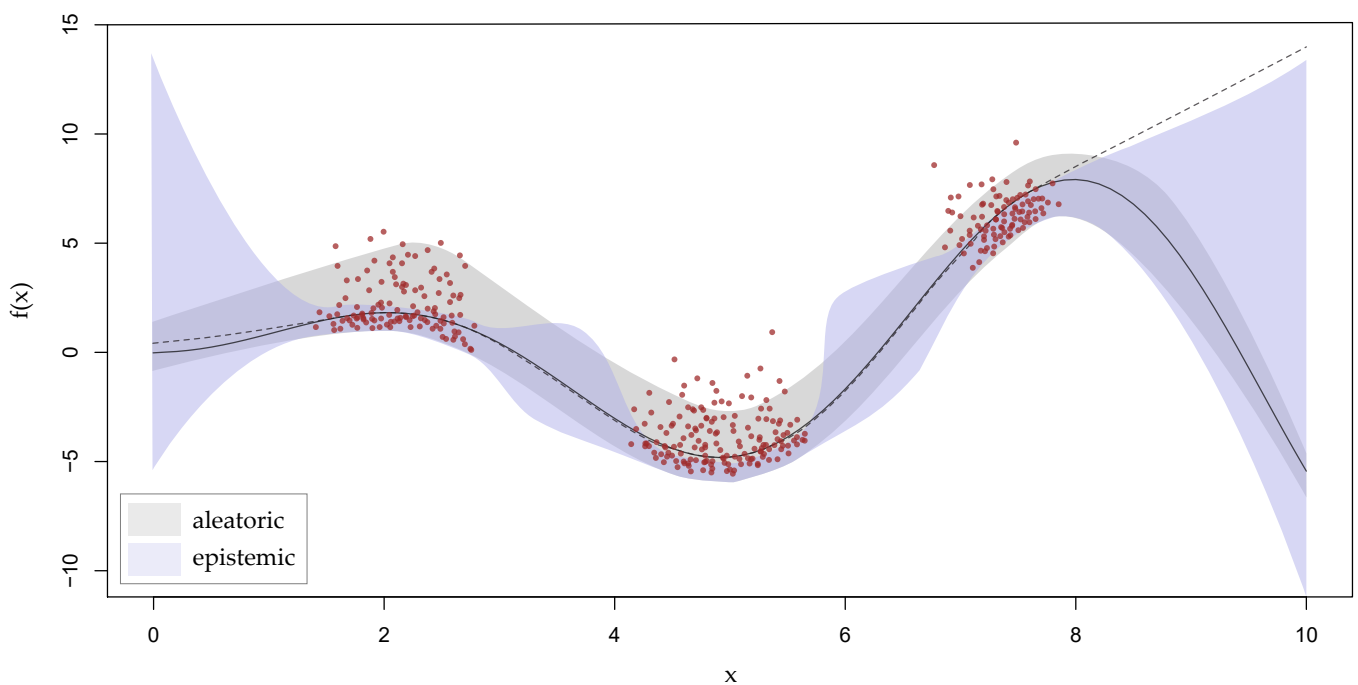

Figure 2: Transect with location of the sampling units in red, the true (solid line) and predicted (dash line) value of the variable of interest, the aleatoric uncertainty (grey shade) and epistemic uncertainty (blue shape). When no observations are present, the epistemic uncertainty increases. The aleatoric uncertainty remains somewhat constant across the transect.

Most studies to date do not provide estimate of the uncertainty (Table 1). Successful attempts have been made by Vaysse \& Lagacherie (2017) and Wadoux (2019b) to report prediction intervals for random forest and neural networks models, respectively. Confidence intervals are reported is several studies (e.g. Hamzehpour et al., 2019; Gomes et al., 2019) and are obtained by training multiple disjoint models using bootstrapped samples of the original data. In a few studies, the variance obtained by bootstrapping is averaged by kriging of the residuals (Viscarra-Rossel et al., 2015). From Fig. 2 it follows that if sampling units are selected from a small area in the feature or geographic space, then there will be little uncertainty in this area. Likewise the uncertainty dramatically increases when areas of the feature space are under-sampled, or even worse, ignored. When sampling units are clustered, (spatial) cross-validation might not be sufficient to define realistic prediction accuracy measures because the sampling units used for validation are taken from similar regions of the feature space while the model is biased towards these same regions (Gahegan, 2000). While the (spatial) cross-validation results might show strong agreement between predicted and measured soil property or class and therefore validate a ML 
model with very high predictive abilities, an uncertainty quantification would show unrealistic predictions characterized by a large uncertainty (see right-hand side of Fig. 2). This is the results of ML algorithm being very poor predictors for extrapolating to areas of the covariate space that is not comprised in the calibration sample. Uncertainty quantification that separates out data and model uncertainties is thus recommended to complete the evaluation of the predicted maps.

We derive a complementary note about the generation of digital soil maps with ML by the private sector. Companies and commercial software usually do not report measures of the uncertainty associated to the maps and there is no transparency requirement on the methods and quality of soil data. Reporting the uncertainty associated to the prediction is essential to guide decision-making and political action. The danger comes from the generated map which gives the appearance of scientific knowledge where there is none. Making a decision made on maps which are presumed correct but are in fact away from reality, is presumably worse than making a decision made in full appreciation of the limits of the map.

\subsection{Validation}

Studies by Roberts et al. (2017) and Ruß \& Brenning (2010) have found that the estimated performance of the machine learning algorithms applied to spatial data depends on the validation strategy. In DSM, model performance is usually assessed using random $k$-fold cross-validation (CV) or single random split of a sample into calibration and validation and/or test subsamples. These strategies give considerably over-optimistic validation statistics estimates because of the presence of autocorrelation in the observations (Micheletti et al., 2014; Gasch et al., 2015; Meyer et al., 2018). Validation statistics estimated from a random split of the master sample assess the ability of the model to reproduce the calibration sample but fail to assess the model performance in terms of spatial mapping (Meyer et al., 2019). As an alternative, several methods (Brenning, 2012; Le Rest et al., 2014; Pohjankukka et al., 2017; Meyer et al., 2019) for spatial cross-validation are proposed to account for spatial autocorrelation of the observations. Two main strategies are adopted. Roberts et al. (2017); Brenning (2012); Meyer et al. (2019) use a spatial block approach for $k$-fold CV where the master sample is divided into $k$ spatially disjoint subsamples using clustering algorithms on the coordinates or by dividing the spatial domain based on $k$ cells. In Le Rest et al. (2014) and Pohjankukka et al. (2017), observations from the calibration subsample that are within a given geographic distance of the validation subsample are omitted from the calibration subsample, after which the model is fitted using the remaining observations from the calibration subsample. While these 
two approaches account for spatial autocorrelation of the observation during validation, further research is required to provide guideline to select the realistic distance from which a validation data point is statistically independent from the calibration sample so as to avoid the opposite effect, i.e. extrapolation and subsequent underoptimistic validation statistics estimates. Spatial-cross validation is integrated in the framework presented in Fig. 3.

Research on spatial cross-validation has drawn attention to the role of autocorrelation on the calibration on the machine learning algorithms. Schratz et al. (2019) show that hyperparameter tuning is also impacted by spatial autocorrelation, and that overoptimistic results are reported when the same data are used for performance assessment and parameter tuning. They proposed a nested (block) cross-validation approach for hyperparameter tuning (Schratz et al., 2019) where spatial block are split a second time into spatially disjoint geographic subsamples used to optimize the hyperparameters. The major disadvantage of this method is the dramatic increase in computing time, which is solved by distributed (parallel) computing solutions. Similarly to the hyperparameter tuning using nested spatial cross-validation, Meyer et al. (2018) showed that autocorrelated covariates lead to overfitting and visible artefacts in the predicted map. The study proposes an iterative procedure for variable selection where a group of two variables is first selected based on the error computed with spatial cross-validation, and new variables are iteratively added only if these increase the model performance. The study of Meyer et al. (2018) gives another argument against the use of covariates describing the spatial dependency as these lead to misinterpretation of the model's important contributors and impossibility for the model to generalize.

Meyer et al. (2019) emphasize the value of visual examination of the predicted maps in addition to the statistical validation. In Meyer et al. (2019), two maps with similar map validation accuracy statistics have a different spatial pattern. The study shows that this is due to the selected covariates, some having strong spatial autocorrelation leading to visible artefacts in the predicted map. This highlights the need for research on the evaluation of predicted maps in terms of spatial pattern. Poggio et al. (2019) compare the spatial structure of predicted versus observed values by computing the area under the curve of variograms fitted on the validation locations for both predicted and observed probability of having a peat soil. This relies, however, on the assumption that the variogram of the validation locations represent the mapped area. More research in this direction will be valuable for future DSM studies. To date, visual assessment of the map to detect artefacts, and in consideration 


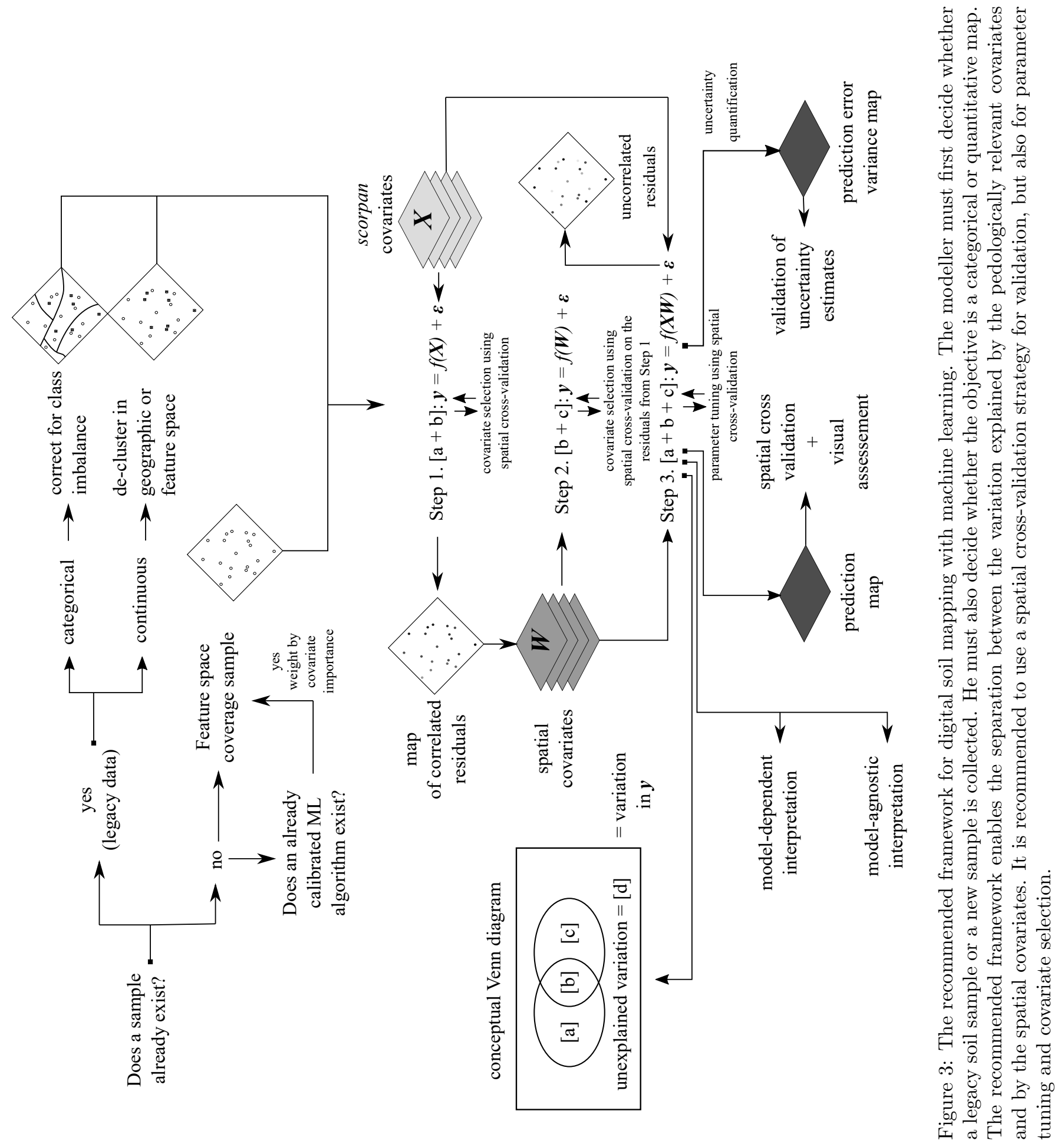


of our knowledge of soil forming processes, is the best option.

\subsection{Machine learning and pedological knowledge}

Accounting for existing expert soil knowledge in DSM with machine learning is a challenging exercise (Ma et al., 2019). ML algorithms do not build on any existing a priori conceptual model of the soil processes and only processes that are conveyed by the input data are represented in the map (Coveney et al., 2016; Koch et al., 2019). To prevent extrapolation, Hengl et al. (2014) do not provide soil maps in some undersampled areas of the globe such as deserts and glaciers for global mapping of several soil properties. This stems from incomplete datasets of soil observations for these areas, despite that extensive expert knowledge exists. In Hengl et al. (2017a) this is solved by integrating the expert knowledge in the form of expert-based pseudo-points to guide the ML model in areas of evident extrapolation. In Koch et al. (2019), 600 pseudo-points are also added in under-represented areas of the geographic space. The study stresses the importance of consulting an expert when building a ML model. In the same study, meaningful covariates are selected based on existing knowledge on the soil process, and plausibility of the predicted soil map is made in consideration of the knowledge of soil forming process. On many occasions, meaningful covariates are selected for mapping soil properties or classes. For example, Brungard et al. (2015) used a set of covariates selected a priori by an expert on the area under study. In Viscarra-Rossel \& Chen (2011) a set of scorpan covariates is selected for mapping soil properties in Australia. These examples show that in the literature, adding expert-based pseudo-points and selecting meaningful covariates are, to date, two straightforward options to include existing knowledge into a ML algorithm for DSM.

The above shows that little is known on how to account for existing knowledge in ML models. Unfortunately, this is the same order in which the complexity of the models increases and our understanding of the model functioning decreases. The increasing caution in the use of predictions made by a complex ML model that one should expect as a result is not evident. A ML model predicting a number based on relationships between covariates that are unknown in the view of existing knowledge, should not be taken with the same seriousness as a number predicted by mechanistic steps or an established theory. Improvement in this situation is made by ensuring that the calibrated ML algorithm matches the existing knowledge of the soil processes, for example by reflecting or confirming the current hypothesis or prior knowledge on the soil spatial variation for an area. If the model prediction does not agree with existing maps, this means that the model has instead modelled a different 
process and is thus likely to be invalid. A model is invalid until it is validated, not only against data, but also against the researcher experience and validation of the model creation process (Gahegan, 2019). In short, pedological knowledge should be integrated to enforce results consistent with the existing scientific principles. This can be done at each step of the model building, calibration and validation. One can incorporate additional knowledge by selecting appropriate covariates or adding pseudo-points. In model building, knowledge takes the form of a hybrid model, a specific model architecture or objective function (in neural networks models) constraining the calibration process according to specific knowledge. For example, Wadoux (2019b) adds the constraint that the prediction of topsoil clay, silt and sand must sum to $100 \%$ in a neural network model. Finally, pedological knowledge is used to make post-hoc checks on the plausibility of the calibrated model and predicted maps.

Gahegan (2019) stress that since ML models (the author used the term "predictive process model" in the sense in which "machine learning models" is used in this article) have no connection to established theory, one can never be sure that the outcome is realistic given the real-world processes involved. The problem is that a non-valid model is difficult to recognize and to reject since it is often not interpretable by a human. To ensure that models fit the existing knowledge, they must be opened and understood in their functioning. Opening the "black box" is then necessary but not straightforward (see next section on interpretability), and is often reduced to the analysis of which environmental covariates are the most often used by the model to make a prediction (see for example Mahmoudabadi et al. (2017) or McNicol et al. (2019)).

Several authors, however, have warned against the use of accuracy metrics for pedological interpretation (e.g. Fourcade et al. (2018) or Wadoux et al. (2019c)). Wadoux et al. (2019c) use meaningless, pseudo-covariates to map soil organic carbon over a hypothetical area. The authors obtain an accurate map, and conclude that ML algorithm should not be used for obtaining new soil knowledge because the ML algorithm aims at predicting a pattern rather than finding causal relationships. Wadoux et al. (2019c) suggest to use calibrated ML models as a "hypothesis discovery" tool, in which the mechanisms conveyed by the calibrated ML model are supplied to the researcher for possible explanations of the soil process, which can then be confronted to experiments and principles of soil genesis. The challenge that then arises, noticed by Gahegan (2019) is the conversion of the mechanisms of the ML model (the model "knowledge") from a data language to a human one. The data language is typically parameters or metrics such as the "mean decrease of purity" 
or "Gini importance index" of a covariate to assess its importance in the prediction of a soil property or class. Such metrics are not interpretable in terms of human explanation and they do not relate to soil processes. Translating the data language to the domain (the human language) requires some attention and further research. More discussion on this issue is found in Gahegan et al. (2001).

\subsection{Interpretation of the models}

Soil scientists rely on ML algorithms to gain insights into the modelled processes. Despite providing higher prediction accuracy than other conventional models, ML models are considered as a black box. Broadly speaking, we do not learn from the model how the input covariates are related to the output soil property or classes, and what are the underlying mechanisms behind the prediction. This is unfortunate for soil science because in many cases the model itself is considered as a source of knowledge in addition to the collected soil data. Scientific findings remain hidden when the model only gives a prediction without explanations. In this case, the interpretability of the model warrants the extraction of the knowledge captured by the calibrated model. Miller (2019) defines intepretability as the degree to which human can understand the cause of a decision. In general, the need for interpretability of a machine learning algorithm stems from a deficiency in problem formalization (DoshiVelez \& Kim, 2017; Molnar, 2019). This means that for a given task (i.e. mapping the spatial distribution of soil organic carbon), the prediction itself does not fully solve the original problem. We suggest three reasons which drive the demand for interpretability in DSM (adapted from Doshi-Velez \& Kim (2017)). The first and most obvious reason is to increase our scientific understanding of the soil system by extracting knowledge from the mechanisms captured by the model. Scientists wish to know which are the drivers of a soil process and, more importantly, whether the mechanisms captured by the model confirm our scientific understanding of the system (see Section 3.7). The second reason is to audit the calibrated ML algorithm. Is the ML algorithm predicting for the right reasons? If a scientist makes a model for mapping the topsoil nitrogen content of a field, the interpretation might reveal that the model is actually predicting soil clay, that is, a proxy of the initial objective. The third reason is to avoid financial loss or to prevent a safety issue. Take the example of the remediation of the soil due to radioactive fallout after the Fukushima nuclear accident. A map of contaminated soils made by a ML algorithm would typically predict the dominant soil type characteristics, i.e. forest soil (about $75 \%$ of the area), for classification into contaminated or not contaminated areas. Interpretation of the model might then reveal that the important features learned by the model are unrealistic for agricultural landscapes and residential areas whose remediation is yet 
critical to safely move back the population (Evrard et al., 2019). 


\section{Humans

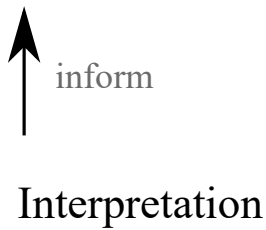

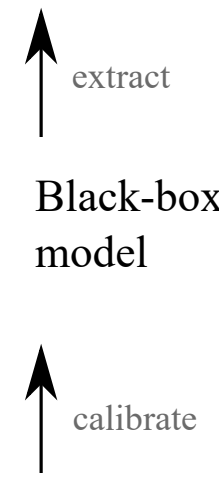

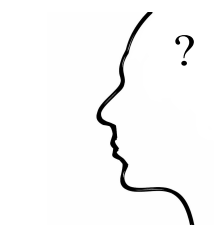

Data $\quad\left(\begin{array}{c}y_{1} \\ y_{2} \\ \vdots \\ y_{m}\end{array}\right)\left(\begin{array}{cccc}x_{1,1} & x_{1,2} & \cdots & x_{1, n} \\ x_{2,1} & x_{2,2} & \cdots & x_{2, n} \\ \vdots & \vdots & \ddots & \vdots \\ x_{m, 1} & x_{m, 2} & \cdots & x_{m, n}\end{array}\right)$
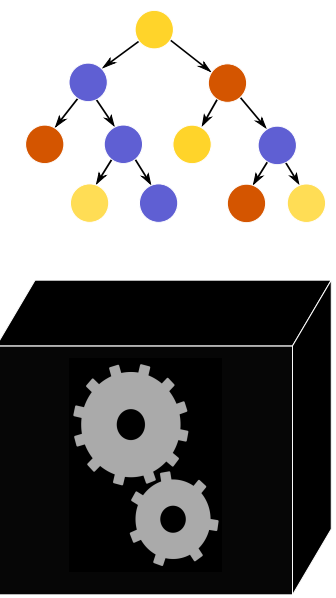

sample

Nature

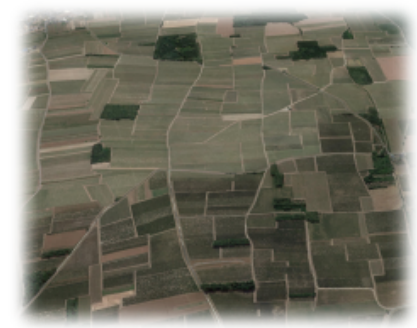

Figure 4: Summary framework for model-agnostic interpretable ML, adapted from Molnar (2019). The lowest level is the reality, the unknown real-world soil that one wants to predict. The second level is the dataset that is extracted from the reality. We collect a fraction of the reality, a sample, and link it to exhaustively known environmental covariates. The relationships between the covariates and the sample is learned by a black-box machine learning model (level 3), on top of which comes the interpretation level to extract some knowledge from the structure of the calibrated model. The structure of the model is converted to human understandable knowledge. 
A straightforward way to increase interpretability is to decrease model complexity, for example by building a single decision tree instead of a random forest composed of several thousand trees. A simple model enables visualization of the important mechanisms of the model and resultant explanations. For DT algorithms, it is possible to map the predicted values for specific rules (if the model is sufficiently simple). Decreasing complexity, however, is done at the expense of model prediction accuracy. For more complex ML algorithms, built-in features allow the user to retrieve the variable importance. In decision-tree like algorithms, the variable importance is derived from the thresholds used for the splits. For neural networks, the output weights associated with the input layer neurons provides an indication of the important features (Gahegan, 2000). One drawback of these techniques is their inability to provide information on whether the covariates have a causal link to the modelled soil property or class, which leads several authors to warn against their use for knowledge discovery (e.g. Fourcade et al., 2018). More importantly, these variable importance metrics are summary statistics not always meaningful and they are model-specific, i.e. they preclude comparison between models or parts of the predicted map. Molnar (2019) reviews techniques to interpret ML algorithms and define two main categories of interpretation techniques. The first are the model specific ones. They are routinely used in DSM activities (e.g. RF variable importance). The second category falls into model-free techniques, also called model-agnostics (Molnar, 2019). It enables the users to use any model, thus not restraining themselves to simple models or models with embedded features of interpretation. A summary of how model-agnostic techniques are employed is shown in Fig. 4. Examples of model-agnostic techniques are the partial dependence plot (Friedman, 2001) if the number of covariate is small (two maximum), individual conditional expectation (Goldstein et al., 2015), and global or local model-agnostic explanation (LIME, Ribeiro et al., 2016). Finally, sensitivity analysis is also a straightforward means of post-hoc interpretation of how the model output depends upon the different covariates.

\section{The way forward}

Machine learning algorithms are now extensively used in soil mapping for regression and classification purposes, much in the same way as routinely employed in other fields of science. There is no doubt that prediction accuracy benefits from these data-driven models because ML algorithms are not constrained by a pre-defined conceptual model of the soil spatial variation, in comparison to mechanistic or even geostatistical models. The question now is how to increase our scientific understanding of the soil and how to adapt and guide the use of ML to the challenges pertaining 
to soil mapping and soil science in general. Future research on soil mapping with machine learning should incorporate the three core elements proposed by Roscher et al. (2019) and Lipton (2018) which we adapted, as follows:

Plausibility: Models should not only be accurate but also valid in light of the current knowledge and scientific theories. A model should predict for the right reasons. The plausibility is the solution path taken by the ML algorithm to link the input to the output, and does not depend directly on the data (Lipton, 2018). In practical terms, it starts with the model building step, by feeding the model with credible covariates and by accounting for the spatial particularities of soil data. Spatial or temporal correlation among data should be modelled, either by using a specific model (e.g. a convolutional neural network), or by using a model architecture that accounts for this particularity (see Section 3.3). Plausibility also takes the form of model constraints, to avoid the prediction of unrealistic proportions or ratios. The plausibility can be further tested in terms of model simulatability (Lipton, 2018). Since ML algorithms can model arbitrary patterns, there should be some attempts to test the model with synthetic data or data from a calibrated mechanistic model representing a large range of dynamics (Reichstein et al., 2019). Increasing model plausibility will facilitate the acceptance of ML to a large range of scenarios in soil science.

Interpretability: Interpretability is the translation of an abstract model or model output into terms understandable by humans (Montavon et al., 2018). Model interpretability pairs with model plausibility and hypothesis discovery. Complex and arbitrary patterns extracted from the data by an algorithm can be understood only by the transparency of the model. Interpretation is obtained by model-specific and model-agnostic methods, described in Section 3.8. Visual examination of the maps is also a means of interpretability. While complex ML models are potentially harmful because they often do not model any real-world process, there is an opportunity to challenge existing knowledge by post-hoc comparison of existing maps produced by expert knowledge with the maps predicted by a ML model, and by analysis of the striking differences. This is possible only if the model is interpretable by humans and the physical relationships between variables are realistic (the model is plausible). Model interpretation is also an opportunity to generate new hypotheses, by interpreting the relationships found by the ML algorithm in the stores of soil data. The new hypotheses derived by these interpretations may challenge existing knowledge on the soil spatial variation and genesis. 
Explainability: Modellers should shy away from mindless model fitting and prediction and intensify research on models that both predict and explain. Explanations aim to answer the three questions: what is the modelled process?, how has it been modelled?, and why has this process been modelled? (Miller, 2019). In this sense, explaining a process is an interpretation of a ML model plus expert knowledge and contextual information. For example, a different explanation is warranted when one wants to explain the pattern of a predicted soil map or the reason for two close predicted soil classes to be different. To explain, the modeller uses the data, the plausibility of the model and its interpretation using expert knowledge (see Fig. 5). Explainability is helped by model structure providing algorithmic explanations in the form of graphs or equations.

An example of model structure providing algorithmic explanations in DSM is found with the use of Bayesian belief networks (BBN, Cooper, 1990) in Mayr et al. (2010) and later Taalab et al. (2015). BBN is a probabilistic graphical model predicting the likely value of a soil property or class given conditional dependencies between covariates. Recent advances in ML have made a step further by discovering the graph structure directly from the data. However, while BNN is an interpretable ML model of conditional dependence between variables, the process that generated these dependencies remains hidden. To discover new processes from data, inductive process modelling (Asgharbeygi et al., 2006) and genetic algorithms (Goldberg \& Holland, 1988) are the way forward. Both are automated model discovery process, in which equations describing a process are inductively (i.e. using the data) assembled into a single predictive model by heuristic search methods (Bridewell et al., 2008; Gahegan, 2019). The calibrated model is a set of equations constrained by existing, verified equations (e.g. differential equations of the water flow) representing causal relationships between variables. The model can be refined using expert knowledge and additional data (Dale et al., 1989). More importantly, these models produce explanations, which can be refuted or approved in light of scientific principles. 


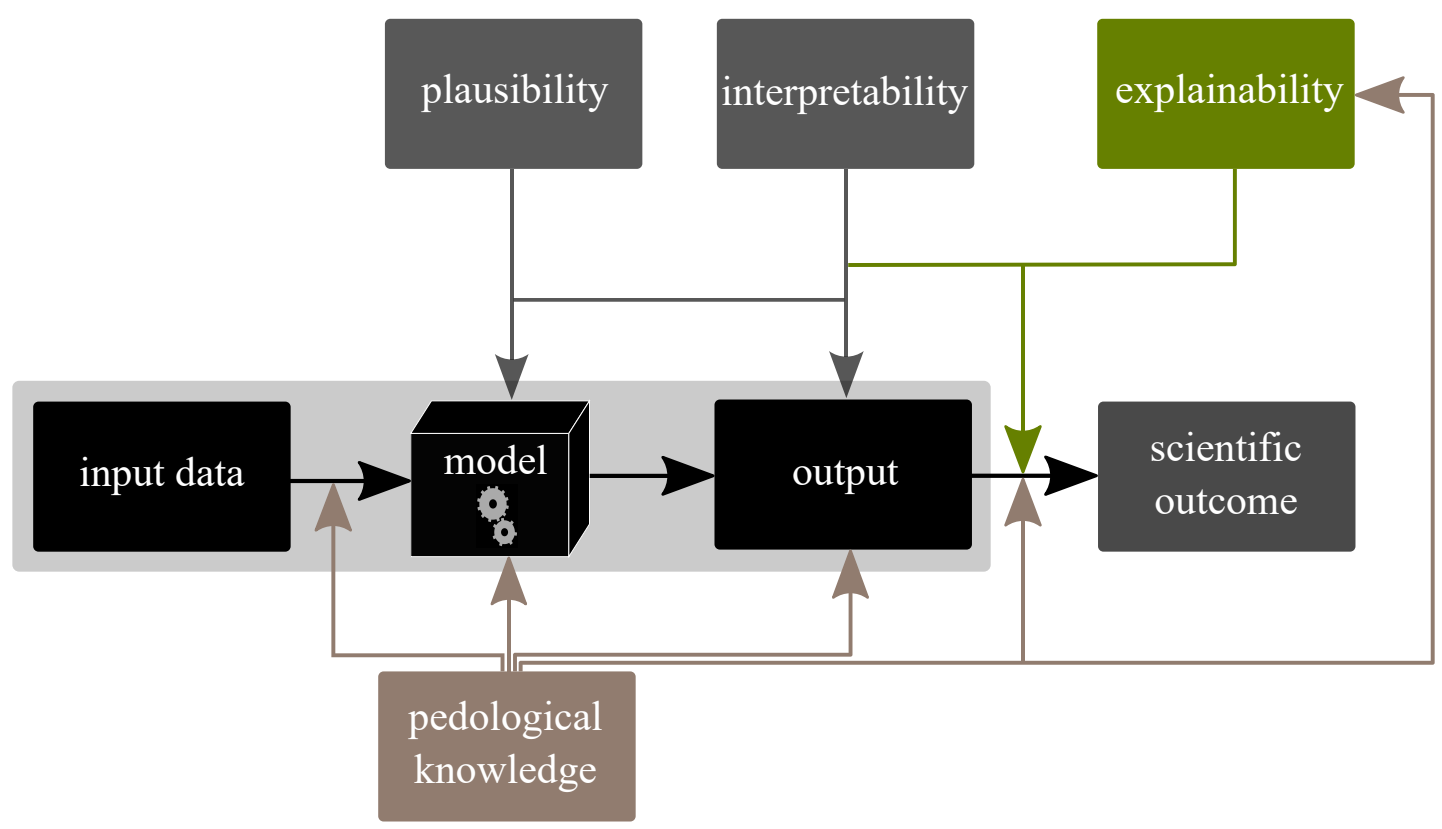

Figure 5: Conceptual framework for the derivation of a scientific outcome from a ML model, adapted from Roscher et al. (2019). The light grey box represents the conventional use of ML algorithms in digital soil mapping, in which an output is derived from a calibrated ML model given a set of input data. A scientific outcome is obtained by explaining the output of a model using pedological knowledge, but also by ensuring scientific consistency at each link of the chain. Alternatively, a plausible and interpretable model can be explained using pedological knowledge.

Figure 5 illustrates the central role played by the three elements plausibility, interpretability and explainability in obtaining a scientific outcome from machine learning. Fig. 5 shows that the three core elements are conditioned to the use of pedological knowledge at each link of the chain. Enforcing pedological knowledge during modelling restricts the solution space to scientifically consistent results and may decrease the overall prediction accuracy. For digital soil mapping purposes, it is not obvious whether an increase of predictive accuracy worth the substantial decrease of model consistency. For this reason, recent studies (e.g. Bennett et al., 2013; Lapuschkin et al., 2019) advocate the use of other criteria to measure the overall performance, such as model complexity or consistency (Karpatne et al., 2017). Including other criteria to assess the overall performance of a ML model would certainly make one step towards "conscious" digital soil mapping, and participate to the uptake of knowledge discovery via machine learning in soil science. 


\section{Conclusion}

In this contribution, we have reviewed the current and prospective use of ML algorithms for digital soil mapping. From the existing use of ML in DSM, we identified key challenges and provided partial solutions We draw the following conclusions.

- There has been a large number of studies mapping soil properties or classes using ML algorithms. A wide range of soil properties, attributes and types have been predicted. Likewise, an increasing number of machine learning algorithms have been tested. Case studies are dominated by the use of legacy samples for local to regional scale (about $10^{4} \mathrm{~km}^{2}$ ) areas. Ensemble of different algorithms to improve prediction are gaining more attention. All studies reported at least one validation statistics but few reported the uncertainty associated with the prediction.

- The configuration of a good sampling design for mapping with machine learning is largely unknown. The impact of the sampling design on model calibration and prediction has generally been disregarded. More research is needed in this direction.

- A large number of studies have focused solely on achieving a high mapping accuracy. Comparison between models and other studies are made based on validation statistics, while ignoring model complexity or consistency with respect to the existing pedological knowledge.

- The benefit of using a large number of covariates, or pseudo-covariates accounting for residual spatial autocorrelation for mapping using ML algorithms should be avoided. To build consistent models, we suggested to select a set of pedologically relevant covariates, and to model the potential residual spatial autocorrelation with post-hoc fitting of another model using spatial surrogate covariates. This procedure also enables a separate analysis of the variation explained by environmental or spatial covariates.

Overall, our review of the literature suggested that in recent studies inference is relegated to the background with the emergence of the mapping accuracy as the sole standard by which progress is measured. While the mapping accuracy is valuable, it should not be the only objective one should pursue. To date, ML is applied to digital soil mapping the same way as other fields such as image detection or pattern recognition do. Any prediction can become a soil map, whether it contains soil knowledge or not, and without any assessment on whether the fitted relationships relate to a 
real-world soil process.

We also found, however, that there is opportunity to include pedological knowledge at each step of the modelling chain, to improve or correct the existing dataset, to design the model architecture, to constrain the model calibration, or to analyse the output using post-hoc checks on the predicted soil maps. Future studies on DSM should use plausible, interpretable and explainable ML models to extract novel scientific results from soil data. One step towards achieving this goal is to integrate model consistency in addition to model prediction accuracy to evaluate the overall performance of the mapping approach. This will ensure that future studies use models that are not only accurate but also valid in light of the current knowledge and scientific theories.

\section{Acknowledgement}

Budiman Minasny is member of a consortium supported by LE STUDIUM Loire Valley Institute for Advanced Studies through its LE STUDIUM Research Consortium Programme.

\section{References}

Adhikari, K., Hartemink, A. E., Minasny, B., Kheir, R. B., Greve, M. B., \& Greve, M. H. (2014). Digital mapping of soil organic carbon contents and stocks in denmark. PLOS ONE, 9, e105519.

Aitkenhead, M. J., \& Coull, M. C. (2016). Mapping soil carbon stocks across Scotland using a neural network model. Geoderma, 262, 187-198.

Akpa, S. I. C., Odeh, I. O. A., Bishop, T. F. A., \& Hartemink, A. E. (2014). Digital mapping of soil particle-size fractions for Nigeria. Soil Science Society of America Journal, 78, 1953-1966.

Arrouays, D., McKenzie, N., Hempel, J., de Forges, A. R., \& McBratney, A. B. (2014). GlobalSoilMap: Basis of the Global Spatial Soil Information System. CRC press, Boca Raton, USA.

Asgharbeygi, N., Langley, P., Bay, S., \& Arrigo, K. (2006). Inductive revision of quantitative process models. Ecological Modelling, 194, 70-79. 
Batjes, N. H., Ribeiro, E., van Oostrum, A., Leenaars, J., Hengl, T., \& Mendes de Jesus, J. (2017). WoSIS: providing standardised soil profile data for the world. Earth System Science Data, 9, 1-14.

Beguin, J., Fuglstad, G.-A., Mansuy, N., \& Paré, D. (2017). Predicting soil properties in the Canadian boreal forest with limited data: Comparison of spatial and nonspatial statistical approaches. Geoderma, 306, 195-205.

Behrens, T., Förster, H., Scholten, T., Steinrücken, U., Spies, E.-D., \& Goldschmitt, M. (2005). Digital soil mapping using artificial neural networks. Journal of Plant Nutrition and Soil Science, 168, 21-33.

Behrens, T., Schmidt, K., MacMillan, R. A., \& Viscarra-Rossel, R. A. (2018a). Multi-scale digital soil mapping with deep learning. Scientific Reports, 8, 15244.

Behrens, T., Schmidt, K., Viscarra-Rossel, R. A., Gries, P., Scholten, T., \& MacMillan, R. A. (2018b). Spatial modelling with Euclidean distance fields and machine learning. European Journal of Soil Science, 69, 757-770.

Behrens, T., Zhu, A.-X., Schmidt, K., \& Scholten, T. (2010). Multi-scale digital terrain analysis and feature selection for digital soil mapping. Geoderma, 155, $175-185$.

Bel, L., Allard, D., Laurent, J. M., Cheddadi, R., \& Bar-Hen, A. (2009). CART algorithm for spatial data: Application to environmental and ecological data. Computational Statistics \& Data Analysis, 53, 3082-3093.

Bel, L., Laurent, J. M., Bar-Hen, A., Allard, D., \& Cheddadi, R. (2005). A spatial extension of CART: application to classification of ecological data. In P. Renard, H. Demougeot-Renard, \& R. Froidevaux (Eds.), Geostatistics for Environmental Applications (pp. 99-109). Springer, Berlin, Heidelberg.

Bennett, N. D., Croke, B. F. W., Guariso, G., Guillaume, J. H. A., Hamilton, S. H., Jakeman, A. J., Marsili-Libelli, S., Newham, L. T. H., Norton, J. P., Perrin, C. et al. (2013). Characterising performance of environmental models. Environmental Modelling \&5 Software, 40, 1-20.

Blanco, C. M. G., Gomez, V. M. B., Crespo, P., \& Ließ, M. (2018). Spatial prediction of soil water retention in a Páramo landscape: Methodological insight into machine learning using random forest. Geoderma, 316, 100-114.

Braben, D. W. (1985). Innovation and academic research. Nature, 316, 401-402. 
Breiman, L. (2017). Classification and Regression Trees. Routledge, New York, USA.

Brenning, A. (2012). Spatial cross-validation and bootstrap for the assessment of prediction rules in remote sensing: The $\mathrm{R}$ package sperrorest. In 2012 International Geoscience and Remote Sensing Symposium (pp. 5372-5375). IEEE.

Bridewell, W., Langley, P., Todorovski, L., \& Džeroski, S. (2008). Inductive process modeling. Machine learning, 71, 1-32.

Brungard, C. W., Boettinger, J. L., Duniway, M. C., Wills, S. A., \& Edwards Jr, T. C. (2015). Machine learning for predicting soil classes in three semi-arid landscapes. Geoderma, 239, 68-83.

Brus, D. J. (2019). Sampling for digital soil mapping: A tutorial supported by R scripts. Geoderma, 338, 464-480.

Bui, E., Henderson, B., \& Viergever, K. (2009). Using knowledge discovery with data mining from the Australian Soil Resource Information System database to inform soil carbon mapping in Australia. Global Biogeochemical Cycles, 23, GB4033.

Bui, E. N., Loughhead, A., \& Corner, R. (1999). Extracting soil-landscape rules from previous soil surveys. Soil Research, 37, 495-508.

Carré, F., McBratney, A. B., \& Minasny, B. (2007). Estimation and potential improvement of the quality of legacy soil samples for digital soil mapping. Geoderma, $141,1-14$.

Chen, S., Mulder, V. L., Martin, M. P., Walter, C., Lacoste, M., Richer-de Forges, A. C., Saby, N. P. A., Loiseau, T., Hu, B., \& Arrouays, D. (2019). Probability mapping of soil thickness by random survival forest at a national scale. Geoderma, $344,184-194$.

Cialella, A. T., Dubayah, R., Lawrence, W., \& Levine, E. (1997). Predicting soil drainage class using remotely sensed and digital elevation data. Photogrammetric Engineering and Remote Sensing, 63, 171-177.

Cooper, G. F. (1990). The computational complexity of probabilistic inference using bayesian belief networks. Artificial intelligence, 42, 393-405.

Coveney, P. V., Dougherty, E. R., \& Highfield, R. R. (2016). Big data need big theory too. Philosophical Transactions of the Royal Society A: Mathematical, Physical and Engineering Sciences, 374, 20160153. 
Cressie, N., \& Johannesson, G. (2008). Fixed rank kriging for very large spatial data sets. Journal of the Royal Statistical Society: Series B (Statistical Methodology), 70, 209-226.

Dai, F., Zhou, Q., Lv, Z., Wang, X., \& Liu, G. (2014). Spatial prediction of soil organic matter content integrating artificial neural network and ordinary kriging in Tibetan Plateau. Ecological Indicators, 45, 184-194.

Dale, M. B., McBratney, A. B., \& Russell, J. S. (1989). On the role of expert systems and numerical taxonomy in soil classification. Journal of Soil Science, 40, 223-234.

De Gruijter, J. J., Brus, D. J., Bierkens, M. F. P., \& Knotters, M. (2006). Sampling for Natural Resource Monitoring. Springer Science \& Business Media, Dordrecht, NL.

Dharumarajan, S., Hegde, R., \& Singh, S. K. (2017). Spatial prediction of major soil properties using Random Forest techniques-A case study in semi-arid tropics of South India. Geoderma Regional, 10, 154-162.

Doshi-Velez, F., \& Kim, B. (2017). Towards a rigorous science of interpretable machine learning. arXiv:1702.08608.

Ellili, Y., Malone, B. P., Michot, D., Minasny, B., Vincent, S., Walter, C., \& Lemercier, B. (2019). Comparing three approaches of spatial disaggregation of legacy soil maps based on DSMART algorithm. SOIL Discussions, .

Evrard, O., Laceby, J. P., \& Nakao, A. (2019). Effectiveness of landscape decontamination following the Fukushima nuclear accident: a review. SOIL, 5, 333-350.

Fick, S. E., \& Hijmans, R. J. (2017). Worldclim 2: new 1-km spatial resolution climate surfaces for global land areas. International Journal of Climatology, 37, $4302-4315$.

Forkuor, G., Hounkpatin, O. K. L., Welp, G., \& Thiel, M. (2017). High resolution mapping of soil properties using remote sensing variables in south-western Burkina Faso: a comparison of machine learning and multiple linear regression models. PLOS ONE, 12, e0170478.

Fourcade, Y., Besnard, A. G., \& Secondi, J. (2018). Paintings predict the distribution of species, or the challenge of selecting environmental predictors and evaluation statistics. Global Ecology and Biogeography, 27, 245-256. 
Friedman, J. H. (2001). Greedy function approximation: a gradient boosting machine. Annals of statistics, 25, 1189-1232.

Gahegan, M. (2000). On the application of inductive machine learning tools to geographical analysis. Geographical Analysis, 32, 113-139.

Gahegan, M. (2019). Fourth paradigm GIScience? prospects for automated discovery and explanation from data. International Journal of Geographical Information Science, 34, 1-21.

Gahegan, M., Wachowicz, M., Harrower, M., \& Rhyne, T.-M. (2001). The integration of geographic visualization with knowledge discovery in databases and geocomputation. Cartography and Geographic Information Science, 28, 29-44.

Gasch, C. K., Hengl, T., Gräler, B., Meyer, H., Magney, T. S., \& Brown, D. J. (2015). Spatio-temporal interpolation of soil water, temperature, and electrical conductivity in 3D+ T: The Cook Agronomy Farm data set. Spatial Statistics, $14,70-90$.

Gascon, F., Bouzinac, C., Thépaut, O., Jung, M., Francesconi, B., Louis, J., Lonjou, V., Lafrance, B., Massera, S., Gaudel-Vacaresse, A. et al. (2017). Copernicus Sentinel-2A calibration and products validation status. Remote Sensing, 9, 584.

Georganos, S., Grippa, T., Gadiaga, A. N., Linard, C., Lennert, M., Vanhuysse, S., Mboga, N. O., Wolff, E., \& Kalogirou, S. (2019). Geographical random forests: A spatial extension of the random forest algorithm to address spatial heterogeneity in remote sensing and population modelling. Geocarto International, 1, 1-12.

Goldberg, D. E., \& Holland, J. H. (1988). Genetic algorithms and machine learning. Kluwer Academic Publishers-Plenum Publishers. Kluwer Academic Publishers.

Goldstein, A., Kapelner, A., Bleich, J., \& Pitkin, E. (2015). Peeking inside the black box: Visualizing statistical learning with plots of individual conditional expectation. Journal of Computational and Graphical Statistics, 24, 44-65.

Gomes, L. C., Faria, R. M., de Souza, E., Veloso, G. V., Schaefer, C. E. G. R., \& Fernandes Filho, E. I. (2019). Modelling and mapping soil organic carbon stocks in Brazil. Geoderma, 340, 337-350.

Grimm, R., Behrens, T., Märker, M., \& Elsenbeer, H. (2008). Soil organic carbon concentrations and stocks on Barro Colorado Island-Digital soil mapping using Random Forests analysis. Geoderma, 146, 102-113. 
Guevara, M., Olmedo, G. F., Stell, E., Yigini, Y., Aguilar Duarte, Y., Arellano Hernández, C., Arévalo, G. E., Arroyo-Cruz, C. E., Bolivar, A., Bunning, S. et al. (2018). No silver bullet for digital soil mapping: country-specific soil organic carbon estimates across Latin America. SOIL, 4, 173-193.

Hamzehpour, N., Shafizadeh-Moghadam, H., \& Valavi, R. (2019). Exploring the driving forces and digital mapping of soil organic carbon using remote sensing and soil texture. CATENA, 182, 104141.

Hansen, M. K., Brown, D. J., Dennison, P. E., Graves, S. A., \& Bricklemyer, R. S. (2009). Inductively mapping expert-derived soil-landscape units within dambo wetland catenae using multispectral and topographic data. Geoderma, 150, 7284 .

Häring, T., Dietz, E., Osenstetter, S., Koschitzki, T., \& Schröder, B. (2012). Spatial disaggregation of complex soil map units: A decision-tree based approach in Bavarian forest soils. Geoderma, 185, 37-47.

Hartmann, J., \& Moosdorf, N. (2012). The new global lithological map database GLiM: A representation of rock properties at the Earth surface. Geochemistry, Geophysics, Geosystems, 13, 1-37.

Hawkins, B. A. (2012). Eight (and a half) deadly sins of spatial analysis. Journal of Biogeography, 39, 1-9.

He, H., \& Garcia, E. A. (2009). Learning from imbalanced data. IEEE Transactions on Knowledge and Data Engineering, 21, 1263-1284.

Henderson, B. L., Bui, E. N., Moran, C. J., \& Simon, D. A. P. (2005). Australia-wide predictions of soil properties using decision trees. Geoderma, 124, 383-398.

Hengl, T., de Jesus, J. M., Heuvelink, G. B. M., Gonzalez, M. R., Kilibarda, M., Blagotić, A., Shangguan, W., Wright, M. N., Geng, X., Bauer-Marschallinger, B. et al. (2017a). SoilGrids250m: Global gridded soil information based on machine learning. PLOS ONE, 12, e0169748.

Hengl, T., de Jesus, J. M., MacMillan, R. A., Batjes, N. H., Heuvelink, G. B. M., Ribeiro, E., Samuel-Rosa, A., Kempen, B., Leenaars, J. G., Walsh, M. G. et al. (2014). SoilGrids $1 \mathrm{~km}$ - global soil information based on automated mapping. PLOS ONE, 9, e105992. 
Hengl, T., Leenaars, J. G. B., Shepherd, K. D., Walsh, M. G., Heuvelink, G. B. M., Mamo, T., Tilahun, H., Berkhout, E., Cooper, M., Fegraus, E. et al. (2017b). Soil nutrient maps of Sub-Saharan Africa: assessment of soil nutrient content at 250 m spatial resolution using machine learning. Nutrient Cycling in Agroecosystems, 109, $77-102$.

Hengl, T., Nussbaum, M., Wright, M. N., Heuvelink, G. B. M., \& Gräler, B. (2018). Random forest as a generic framework for predictive modeling of spatial and spatiotemporal variables. PeerJ, 6, e5518.

Heung, B., Ho, H. C., Zhang, J., Knudby, A., Bulmer, C. E., \& Schmidt, M. G. (2016). An overview and comparison of machine-learning techniques for classification purposes in digital soil mapping. Geoderma, 265, 62-77.

Heuvelink, G. B. M., \& Webster, R. (2001). Modelling soil variation: past, present, and future. Geoderma, 100, 269-301.

Holmes, K. W., Odgers, N. P., Griffin, E. A., \& van Gool, D. (2014). Spatial disaggregation of conventional soil mapping across Western Australia using DSMART. In D. Arrouays, N. McKenzie, J. Hempel, A. Richer de Forges, \& A. B. McBratney (Eds.), GlobalSoilMap: Basis of the Global Spatial Soil Information System (pp. 273-279). Taylor \& Francis, London, UK.

Hounkpatin, K. O. L., Schmidt, K., Stumpf, F., Forkuor, G., Behrens, T., Scholten, T., Amelung, W., \& Welp, G. (2018). Predicting reference soil groups using legacy data: A data pruning and Random Forest approach for tropical environment (Dano catchment, Burkina Faso). Scientific Reports, 8, 9959.

Illés, G., Sutikno, S., Szatmári, G., Sandhyavitri, A., Pásztor, L., Kristijono, A., Molnár, G., Yusa, M., \& Székely, B. (2019). Facing the peat $\mathrm{co}_{2}$ threat: digital mapping of Indonesian peatlands - a proposed methodology and its application. Journal of Soils and Sediments, (pp. 1-16).

Jenny, H. (1941). Factors of Soil Formation: A System of Quantitative Pedology. McGrawHill, New York, USA.

Jiang, Z., Li, Y., Shekhar, S., Rampi, L., \& Knight, J. (2017). Spatial ensemble learning for heterogeneous geographic data with class ambiguity: A summary of results. In Proceedings of the 25th ACM SIGSPATIAL International Conference on Advances in Geographic Information Systems 23 (pp. 1-10). ACM. 
Kalambukattu, J. G., Kumar, S., \& Raj, R. A. (2018). Digital soil mapping in a Himalayan watershed using remote sensing and terrain parameters employing artificial neural network model. Environmental Earth Sciences, 77, 203.

Karpatne, A., Atluri, G., Faghmous, J. H., Steinbach, M., Banerjee, A., Ganguly, A., Shekhar, S., Samatova, N., \& Kumar, V. (2017). Theory-guided data science: A new paradigm for scientific discovery from data. IEEE Transactions on Knowledge and Data Engineering, 29, 2318-2331.

Keskin, H., Grunwald, S., \& Harris, W. G. (2019). Digital mapping of soil carbon fractions with machine learning. Geoderma, 339, 40-58.

Kheir, R. B., Greve, M. H., Abdallah, C., \& Dalgaard, T. (2010a). Spatial soil zinc content distribution from terrain parameters: A GIS-based decision-tree model in Lebanon. Environmental Pollution, 158, 520-528.

Kheir, R. B., Greve, M. H., Bøcher, P. K., Greve, M. B., Larsen, R., \& McCloy, K. (2010b). Predictive mapping of soil organic carbon in wet cultivated lands using classification-tree based models: The case study of Denmark. Journal of Environmental Management, 91, 1150-1160.

Kirkwood, C., Cave, M., Beamish, D., Grebby, S., \& Ferreira, A. (2016). A machine learning approach to geochemical mapping. Journal of Geochemical Exploration, $167,49-61$.

Koch, J., Stisen, S., Refsgaard, J. C., Ernstsen, V., Jakobsen, P. R., \& Højberg, A. L. (2019). Modeling Depth of the Redox Interface at High Resolution at National Scale Using Random Forest and Residual Gaussian Simulation. Water Resources Research, 55, 1451-1469.

Kovačević, M., Bajat, B., \& Gajić, B. (2010). Soil type classification and estimation of soil properties using support vector machines. Geoderma, 154, 340-347.

Kühn, I., \& Dormann, C. F. (2012). Less than eight (and a half) misconceptions of spatial analysis. Journal of Biogeography, 39, 995-998.

Kühn, I., Nobis, M. P., \& Durka, W. (2009). Combining spatial and phylogenetic eigenvector filtering in trait analysis. Global Ecology and Biogeography, 18, 745758. 
Lacoste, M., Minasny, B., McBratney, A., Michot, D., Viaud, V., \& Walter, C. (2014). High resolution 3d mapping of soil organic carbon in a heterogeneous agricultural landscape. Geoderma, 213, 296-311.

Lagacherie, P. (2008). Digital soil mapping: a state of the art. In A. E. Hartemink, A. McBratney, \& M. de Lourdes Mendonça-Santos (Eds.), Digital Soil Mapping with Limited Data (pp. 3-14). Springer, Dordrecht, Netherlands.

Lagacherie, P., \& Holmes, S. (1997). Addressing geographical data errors in a classification tree for soil unit prediction. International Journal of Geographical Information Science, 11, 183-198.

Lagacherie, P., \& McBratney, A. (2006). Spatial soil information systems and spatial soil inference systems: perspectives for digital soil mapping. Developments in Soil Science, 31, 3-22.

Lamichhane, S., Kumar, L., \& Wilson, B. (2019). Digital soil mapping algorithms and covariates for soil organic carbon mapping and their implications: A review. Geoderma, (pp. 395-413).

Lapuschkin, S., Wäldchen, S., Binder, A., Montavon, G., Samek, W., \& Müller, K.R. (2019). Unmasking Clever Hans predictors and assessing what machines really learn. Nature Communications, 10, 1096.

Le Rest, K., Pinaud, D., Monestiez, P., Chadoeuf, J., \& Bretagnolle, V. (2014). Spatial leave-one-out cross-validation for variable selection in the presence of spatial autocorrelation. Global Ecology and Biogeography, 23, 811-820.

Ließ, M., Glaser, B., \& Huwe, B. (2012). Uncertainty in the spatial prediction of soil texture: comparison of regression tree and random forest models. Geoderma, $170,70-79$.

Ließ, M., Schmidt, J., \& Glaser, B. (2016). Improving the spatial prediction of soil organic carbon stocks in a complex tropical mountain landscape by methodological specifications in machine learning approaches. PLOS ONE, 11, e0153673.

Lipton, Z. C. (2018). The mythos of model interpretability. Queue, 16, 31-57.

Liu, F., Zhang, G.-L., Song, X., Li, D., Zhao, Y., Yang, J., Wu, H., \& Yang, F. (2019). High-resolution and three-dimensional mapping of soil texture of China. Geoderma, 361, 114061. 
Lorenzetti, R., Barbetti, R., Fantappiè, M., L'Abate, G., \& Costantini, E. A. (2015). Comparing data mining and deterministic pedology to assess the frequency of wrb reference soil groups in the legend of small scale maps. Geoderma, 237, 237-245.

Ma, Y., Minasny, B., Malone, B. P., \& Mcbratney, A. B. (2019). Pedology and digital soil mapping (DSM). European Journal of Soil Science, 70, 216-235.

MacKay, D. J. C. (1992). Information-based objective functions for active data selection. Neural Computation, 4, 590-604.

Mahmoudabadi, E., Karimi, A., Haghnia, G. H., \& Sepehr, A. (2017). Digital soil mapping using remote sensing indices, terrain attributes, and vegetation features in the rangelands of northeastern Iran. Environmental Monitoring and Assessment, 189,500 .

Malone, B. P., McBratney, A. B., Minasny, B., \& Laslett, G. M. (2009). Mapping continuous depth functions of soil carbon storage and available water capacity. Geoderma, 154, 138-152.

Mansuy, N., Thiffault, E., Paré, D., Bernier, P., Guindon, L., Villemaire, P., Poirier, V., \& Beaudoin, A. (2014). Digital mapping of soil properties in Canadian managed forests at $250 \mathrm{~m}$ of resolution using the k-nearest neighbor method. Geoderma, 235, 59-73.

Marchant, B. P., Viscarra Rossel, R. A., \& Webster, R. (2013). Fluctuations in method-of-moments variograms caused by clustered sampling and their elimination by declustering and residual maximum likelihood estimation. European Journal of Soil Science, 64, 401-409.

Massawe, B. H. J., Subburayalu, S. K., Kaaya, A. K., Winowiecki, L., \& Slater, B. K. (2018). Mapping numerically classified soil taxa in Kilombero valley, Tanzania using machine learning. Geoderma, 311, 143-148.

Mayr, T., Rivas-Casado, M., Bellamy, P., Palmer, R., Zawadzka, J., \& Corstanje, R. (2010). Two methods for using legacy data in digital soil mapping. In M. A. H. A. K.-B. S. Boettinger J.L., Howell D.W. (Ed.), Digital Soil Mapping (pp. 191-202). Springer, Dordrecht, NL.

McBratney, A. B., Santos, M. M., \& Minasny, B. (2003). On digital soil mapping. Geoderma, 117, 3-52. 
McNicol, G., Bulmer, C., D'Amore, D., Sanborn, P., Saunders, S., Giesbrecht, I., Arriola, S. G., Bidlack, A., Butman, D., \& Buma, B. (2019). Large, climatesensitive soil carbon stocks mapped with pedology-informed machine learning in the North Pacific coastal temperate rainforest. Environmental Research Letters, 14, 014004 .

Meyer, H., Reudenbach, C., Hengl, T., Katurji, M., \& Nauss, T. (2018). Improving performance of spatio-temporal machine learning models using forward feature selection and target-oriented validation. Environmental Modelling 85 Software, $101,1-9$.

Meyer, H., Reudenbach, C., Wöllauer, S., \& Nauss, T. (2019). Importance of spatial predictor variable selection in machine learning applications-moving from data reproduction to spatial prediction. Ecological Modelling, 411, 108815.

Micheletti, N., Foresti, L., Robert, S., Leuenberger, M., Pedrazzini, A., Jaboyedoff, M., \& Kanevski, M. (2014). Machine learning feature selection methods for landslide susceptibility mapping. Mathematical Geosciences, 46, 33-57.

Miller, B. A., Koszinski, S., Wehrhan, M., \& Sommer, M. (2015a). Comparison of spatial association approaches for landscape mapping of soil organic carbon stocks. Soil, 1, 217-233.

Miller, B. A., Koszinski, S., Wehrhan, M., \& Sommer, M. (2015b). Impact of multiscale predictor selection for modeling soil properties. Geoderma, 239, 97-106.

Miller, T. (2019). Explanation in artificial intelligence: Insights from the social sciences. Artificial Intelligence, 267, 1-38.

Mira, M., Weiss, M., Baret, F., Courault, D., Hagolle, O., Gallego-Elvira, B., \& Olioso, A. (2015). The MODIS (collection V006) BRDF/albedo product MCD43D: Temporal course evaluated over agricultural landscape. Remote Sensing of Environment, 170, 216-228.

Mirza, M., \& Osindero, S. (2014). Conditional generative adversarial nets. arXiv: 1411.1784 .

Molnar, C. (2019). Interpretable machine learning. Lulu, Morrisville, USA.

Montavon, G., Samek, W., \& Müller, K.-R. (2018). Methods for interpreting and understanding deep neural networks. Digital Signal Processing, 73, 1-15. 
Moran, C. J., \& Bui, E. N. (2002). Spatial data mining for enhanced soil map modelling. International Journal of Geographical Information Science, 16, 533549 .

Mosleh, Z., Salehi, M. H., Jafari, A., Borujeni, I. E., \& Mehnatkesh, A. (2016). The effectiveness of digital soil mapping to predict soil properties over low-relief areas. Environmental Monitoring and Assessment, 188, 195.

Mulder, V. L., Lacoste, M., Richer-de Forges, A. C., Martin, M. P., \& Arrouays, D. (2016). National versus global modelling the 3D distribution of soil organic carbon in mainland France. Geoderma, 263, 16-34.

Nauman, T. W., \& Duniway, M. C. (2019). Relative prediction intervals reveal larger uncertainty in $3 \mathrm{D}$ approaches to predictive digital soil mapping of soil properties with legacy data. Geoderma, 347, 170-184.

Nussbaum, M., Spiess, K., Baltensweiler, A., Grob, U., Keller, A., Greiner, L., Schaepman, M. E., \& Papritz, A. (2018). Evaluation of digital soil mapping approaches with large sets of environmental covariates. Soil, 4, 1-22.

Odgers, N. P., Sun, W., McBratney, A. B., Minasny, B., \& Clifford, D. (2014). Disaggregating and harmonising soil map units through resampled classification trees. Geoderma, 214, 91-100.

Oldeman, L. R., \& Van Engelen, V. W. P. (1993). A world soils and terrain digital database (SOTER) - An improved assessment of land resources. Geoderma, 60, 309-325.

Oliver, M. A. (1987). Geostatistics and its application to soil science. Soil Use and Management, 3, 8-20.

Ottoy, S., De Vos, B., Sindayihebura, A., Hermy, M., \& Van Orshoven, J. (2017). Assessing soil organic carbon stocks under current and potential forest cover using digital soil mapping and spatial generalisation. Ecological Indicators, 77, 139-150.

Padarian, J., Minasny, B., \& McBratney, A. B. (2019). Using deep learning for digital soil mapping. Soil, 5, 79-89.

Pahlavan-Rad, M. R., \& Akbarimoghaddam, A. (2018). Spatial variability of soil texture fractions and $\mathrm{pH}$ in a flood plain (case study from eastern Iran). CATENA, $160,275-281$. 
Peres-Neto, P. R., Legendre, P., Dray, S., \& Borcard, D. (2006). Variation partitioning of species data matrices: estimation and comparison of fractions. Ecology, 87, $2614-2625$.

Poggio, L., Lassauce, A., \& Gimona, A. (2019). Modelling the extent of northern peat soil and its uncertainty with sentinel: Scotland as example of highly cloudy region. Geoderma, 346, 63-74.

Pohjankukka, J., Pahikkala, T., Nevalainen, P., \& Heikkonen, J. (2017). Estimating the prediction performance of spatial models via spatial k-fold cross validation. International Journal of Geographical Information Science, 31, 2001-2019.

Pouladi, N., Møller, A. B., Tabatabai, S., \& Greve, M. H. (2019). Mapping soil organic matter contents at field level with cubist, random forest and kriging. Geoderma, 342, 85-92.

Pozdnoukhov, A., \& Kanevski, M. (2006). Monitoring network optimisation for spatial data classification using support vector machines. International Journal of Environment and Pollution, 28, 465-484.

Rahman, R., Otridge, J., \& Pal, R. (2017). IntegratedMRF: random forest-based framework for integrating prediction from different data types. Bioinformatics, 33, 1407-1410.

Ramcharan, A., Hengl, T., Nauman, T., Brungard, C., Waltman, S., Wills, S., \& Thompson, J. (2018). Soil property and class maps of the conterminous United States at 100-meter spatial resolution. Soil Science Society of America Journal, 82, 186-201.

Reichstein, M., Camps-Valls, G., Stevens, B., Jung, M., Denzler, J., Carvalhais, N. et al. (2019). Deep learning and process understanding for data-driven earth system science. Nature, 566, 195-204.

Ribeiro, M. T., Singh, S., \& Guestrin, C. (2016). Why should I trust you?: Explaining the predictions of any classifier. In Proceedings of the 22nd ACM SIGKDD International Conference on Knowledge Discovery and Data Mining (pp. 11351144). ACM.

Roberts, D. R., Bahn, V., Ciuti, S., Boyce, M. S., Elith, J., Guillera-Arroita, G., Hauenstein, S., Lahoz-Monfort, J. J., Schröder, B., Thuiller, W. et al. (2017). Cross-validation strategies for data with temporal, spatial, hierarchical, or phylogenetic structure. Ecography, 40, 913-929. 
Roscher, R., Bohn, B., Duarte, M. F., \& Garcke, J. (2019). Explainable machine learning for scientific insights and discoveries. arXiv:1905.08883.

Rudiyanto, B., Minasny, Setiawan, B. I., Saptomo, S. K., McBratney, A. B. et al. (2018). Open digital mapping as a cost-effective method for mapping peat thickness and assessing the carbon stock of tropical peatlands. Geoderma, 313, 25-40.

Ruß, G., \& Brenning, A. (2010). Data mining in precision agriculture: management of spatial information. In International Conference on Information Processing and Management of Uncertainty in Knowledge-Based Systems (pp. 350-359). Springer.

Schratz, P., Muenchow, J., Iturritxa, E., Richter, J., \& Brenning, A. (2019). Hyperparameter tuning and performance assessment of statistical and machine-learning algorithms using spatial data. Ecological Modelling, 406, 109-120.

Scull, P., Franklin, J., \& Chadwick, O. A. (2005). The application of classification tree analysis to soil type prediction in a desert landscape. Ecological Modelling, $181,1-15$.

Segal, M., \& Xiao, Y. (2011). Multivariate random forests. Wiley Interdisciplinary Reviews: Data Mining and Knowledge Discovery, 1, 80-87.

Sergeev, A., Buevich, A., Baglaeva, E., \& Shichkin, A. (2019). Combining spatial autocorrelation with machine learning increases prediction accuracy of soil heavy metals. CATENA, 174, 425-435.

Sharififar, A., Sarmadian, F., Malone, B. P., \& Minasny, B. (2019). Addressing the issue of digital mapping of soil classes with imbalanced class observations. Geoderma, 350, 84-92.

Shi, J., Yang, L., Zhu, A., Qin, C., Liang, P., Zeng, C., Pei, T. et al. (2018). Machinelearning variables at different scales vs. knowledge-based variables for mapping multiple soil properties. Soil Science Society of America Journal, 82, 645-656.

Siewert, M. B. (2018). High-resolution digital mapping of soil organic carbon in permafrost terrain using machine learning: a case study in a sub-Arctic peatland environment. Biogeosciences, 15, 1663-1682.

da Silva Chagas, C., de Carvalho Junior, W., Bhering, S. B., \& Calderano Filho, B. (2016). Spatial prediction of soil surface texture in a semiarid region using random forest and multiple linear regressions. CATENA, 139, 232-240. 
Sinha, P., Gaughan, A. E., Stevens, F. R., Nieves, J. J., Sorichetta, A., \& Tatem, A. J. (2019). Assessing the spatial sensitivity of a random forest model: Application in gridded population modeling. Computers, Environment and Urban Systems, 75, $132-145$.

Somarathna, P. D. S. N., Malone, B. P., \& Minasny, B. (2016). Mapping soil organic carbon content over New South Wales, Australia using local regression kriging. Geoderma regional, 7, 38-48.

Somarathna, P. D. S. N., Minasny, B., \& Malone, B. P. (2017). More data or a better model? figuring out what matters most for the spatial prediction of soil carbon. Soil Science Society of America Journal, 81, 1413-1426.

Song, X.-D., Wu, H.-Y., Ju, B., Liu, F., Yang, F., Li, D.-C., Zhao, Y.-G., Yang, J.L., \& Zhang, G.-L. (2020). Pedoclimatic zone-based three-dimensional soil organic carbon mapping in China. Geoderma, 363, 114145.

Song, Y.-Q., Zhao, X., Su, H.-Y., Li, B., Hu, Y.-M., \& Cui, X.-S. (2018). Predicting spatial variations in soil nutrients with hyperspectral remote sensing at regional scale. Sensors, 18, 3086.

Stojanova, D., Ceci, M., Appice, A., Malerba, D., \& Džeroski, S. (2013). Dealing with spatial autocorrelation when learning predictive clustering trees. Ecological Informatics, 13, 22-39.

Stumpf, F., Schmidt, K., Behrens, T., Schönbrodt-Stitt, S., Buzzo, G., Dumperth, C., Wadoux, A., Xiang, W., \& Scholten, T. (2016). Incorporating limited field operability and legacy soil samples in a hypercube sampling design for digital soil mapping. Journal of Plant Nutrition and Soil Science, 179, 499-509.

Subburayalu, S. K., \& Slater, B. K. (2013). Soil series mapping by knowledge discovery from an Ohio county soil map. Soil Science Society of America Journal, 77, $1254-1268$.

Szatmári, G., \& Pásztor, L. (2019). Comparison of various uncertainty modelling approaches based on geostatistics and machine learning algorithms. Geoderma, 337, 1329-1340.

Szatmári, G., Pirkó, B., Koós, S., Laborczi, A., Bakacsi, Z., Szabó, J., \& Pásztor, L. (2019). Spatio-temporal assessment of topsoil organic carbon stock change in Hungary. Soil and Tillage Research, 195, 104410. 
Taalab, K., Corstanje, R., Mayr, T., Whelan, M., \& Creamer, R. (2015). The application of expert knowledge in bayesian networks to predict soil bulk density at the landscape scale. European Journal of Soil Science, 66, 930-941.

Taghizadeh-Mehrjardi, R., Minasny, B., Sarmadian, F., \& Malone, B. P. (2014). Digital mapping of soil salinity in Ardakan region, central Iran. Geoderma, 213, $15-28$.

Taghizadeh-Mehrjardi, R., Minasny, B., Toomanian, N., Zeraatpisheh, M., AmirianChakan, A., \& Triantafilis, J. (2019a). Digital mapping of soil classes using ensemble of models in Isfahan region, Iran. Soil Systems, 3, 37.

Taghizadeh-Mehrjardi, R., Nabiollahi, K., \& Kerry, R. (2016). Digital mapping of soil organic carbon at multiple depths using different data mining techniques in Baneh region, Iran. Geoderma, 266, 98-110.

Taghizadeh-Mehrjardi, R., Schmidt, K., Eftekhari, K., Behrens, T., Jamshidi, M., Davatgaar, N., Toomanian, N., \& Scholten, T. (2019b). Synthetic resampling strategies and machine learning for digital soil mapping in Iran. European Journal of Soil Science, .

Taghizadeh-mehrjardi, R., Toomanian, N., Khavaninzadeh, A. R., Jafari, A., \& Triantafilis, J. (2016). Predicting and mapping of soil particle-size fractions with adaptive neuro-fuzzy inference and ant colony optimization in central Iran. European Journal of Soil Science, 67, 707-725.

Tajik, S., Ayoubi, S., Shirani, H., \& Zeraatpisheh, M. (2019). Digital mapping of soil invertebrates using environmental attributes in a deciduous forest ecosystem. Geoderma, 353, 252-263.

Tuia, D., Pozdnoukhov, A., Foresti, L., \& Kanevski, M. (2013). Active learning for monitoring network optimization. In J. Mateu, \& W. G. Müller (Eds.), SpatioTemporal Design: Advances in Efficient Data Acquisition (pp. 285-318). Wiley Online Library, Chichester, UK.

Tziachris, P., Aschonitis, V., Chatzistathis, T., \& Papadopoulou, M. (2019). Assessment of spatial hybrid methods for predicting soil organic matter using DEM derivatives and soil parameters. CATENA, 174, 206-216.

Uria, B., Côté, M.-A., Gregor, K., Murray, I., \& Larochelle, H. (2016). Neural autoregressive distribution estimation. The Journal of Machine Learning Research, $17,7184-7220$. 
Vaysse, K., \& Lagacherie, P. (2015). Evaluating digital soil mapping approaches for mapping GlobalSoilMap soil properties from legacy data in Languedoc-Roussillon (France). Geoderma Regional, 4, 20-30.

Vaysse, K., \& Lagacherie, P. (2017). Using quantile regression forest to estimate uncertainty of digital soil mapping products. Geoderma, 291, 55-64.

Vermeulen, D., \& Van Niekerk, A. (2017). Machine learning performance for predicting soil salinity using different combinations of geomorphometric covariates. Geoderma, 299, 1-12.

Vincent, S., Lemercier, B., Berthier, L., \& Walter, C. (2018). Spatial disaggregation of complex soil map units at the regional scale based on soil-landscape relationships. Geoderma, 311, 130-142.

Viscarra-Rossel, R. A., \& Chen, C. (2011). Digitally mapping the information content of visible-near infrared spectra of surficial Australian soils. Remote Sensing of Environment, 115, 1443-1455.

Viscarra-Rossel, R. A., Chen, C., Grundy, M. J., Searle, R., Clifford, D., \& Campbell, P. H. (2015). The Australian three-dimensional soil grid: Australia's contribution to the GlobalSoilMap project. Soil Research, 53, 845-864.

Wadoux, A. M. J.-C. (2019a). Sampling design optimization for geostatistical modelling and prediction. Ph.D. thesis Wageningen University \& Research.

Wadoux, A. M. J.-C. (2019b). Using deep learning for multivariate mapping of soil with quantified uncertainty. Geoderma, 351, 59-70.

Wadoux, A. M. J.-C., Brus, D. J., \& Heuvelink, G. B. M. (2019a). Sampling design optimization for soil mapping with random forest. Geoderma, 355, 113913.

Wadoux, A. M. J.-C., Padarian, J., \& Minasny, B. (2019b). Multi-source data integration for soil mapping using deep learning. Soil, 5, 107-119.

Wadoux, A. M. J.-C., Samuel-Rosa, A., Poggio, L., \& Mulder, V. L. (2019c). A note on knowledge discovery and machine learning in digital soil mapping. European Journal of Soil Science, .

Wang, B., Waters, C., Orgill, S., Gray, J., Cowie, A., Clark, A., \& Li Liu, D. (2018). High resolution mapping of soil organic carbon stocks using remote sensing variables in the semi-arid rangelands of eastern Australia. Science of The Total Environment, 630, 367-378. 
Wang, S., Zhuang, Q., Wang, Q., Jin, X., \& Han, C. (2017). Mapping stocks of soil organic carbon and soil total nitrogen in Liaoning Province of China. Geoderma, $305,250-263$.

Were, K., Bui, D. T., Dick, Ø. B., \& Singh, B. R. (2015). A comparative assessment of support vector regression, artificial neural networks, and random forests for predicting and mapping soil organic carbon stocks across an Afromontane landscape. Ecological Indicators, 52, 394-403.

Wiesmeier, M., Barthold, F., Blank, B., \& Kögel-Knabner, I. (2011). Digital mapping of soil organic matter stocks using random forest modeling in a semi-arid steppe ecosystem. Plant and Soil, 340, 7-24.

Wu, J., Teng, Y., Chen, H., \& Li, J. (2016). Machine-learning models for on-site estimation of background concentrations of arsenic in soils using soil formation factors. Journal of Soils and Sediments, 16, 1787-1797.

Xu, S., An, X., Qiao, X., Zhu, L., \& Li, L. (2013). Multi-output least-squares support vector regression machines. Pattern Recognition Letters, 34, 1078-1084.

Yamazaki, D., Ikeshima, D., Tawatari, R., Yamaguchi, T., O'Loughlin, F., Neal, J. C., Sampson, C. C., Kanae, S., \& Bates, P. D. (2017). A high-accuracy map of global terrain elevations. Geophysical Research Letters, 44, 5844-5853.

Yang, R.-M., Zhang, G.-L., Liu, F., Lu, Y.-Y., Yang, F., Yang, F., Yang, M., Zhao, Y.-G., \& Li, D.-C. (2016). Comparison of boosted regression tree and random forest models for mapping topsoil organic carbon concentration in an alpine ecosystem. Ecological Indicators, 60, 870-878.

Zeraatpisheh, M., Ayoubi, S., Jafari, A., \& Finke, P. (2017). Comparing the efficiency of digital and conventional soil mapping to predict soil types in a semi-arid region in Iran. Geomorphology, 285, 186-204.

Zeraatpisheh, M., Ayoubi, S., Jafari, A., Tajik, S., \& Finke, P. (2019). Digital mapping of soil properties using multiple machine learning in a semi-arid region, central Iran. Geoderma, 338, 445-452.

Zhu, M., Feng, Q., Zhang, M., Liu, W., Deo, R. C., Zhang, C., \& Yang, L. (2019). Soil organic carbon in semiarid alpine regions: the spatial distribution, stock estimation, and environmental controls. Journal of Soils and Sediments, 19, 1-15. 